\title{
Synergy between Noise Reduction Techniques Applied in Different Industries: A Review
}

\author{
M Yadegari', F Ommi' ${ }^{1 *}$ Z Saboohi ${ }^{2}$ \\ 1. Tarbiat Modares University (TMU), Tehran, Iran \\ 2. A \& S Institute, Ministry of Science, Research, and \\ Technology, Tehran, Iran
}

\begin{abstract}
This review aims to assist aerodynamic noise in three industrial fields: Aerospace, Turbomachinery and Automotive. In this review the general terms in aeroacoustics is defined; and aerodynamic noise sources are recognized. The paper also reviews the brief literature on noise reduction techniques, with a particular focus on the state of the art numerical and experimental works. In Addition, developments in low speed designs for aerodynamic noise reduction, some passive and active methods for example serrated boundaries, porous media, noise absorber, and etc. are discussed. By investigating similarities in noise reduction techniques between these industries, this paper offers an outlook for noise reduction techniques in future.
\end{abstract}

\section{INTRODUCTION}

Sound is defined as Oscillation in pressure, stress, particle movement, particle velocity, etc., generated in a medium with internal forces (e.g., elastic or viscous) and auditory sensation elicited by this oscillation [1]. Unwanted sound, or noise, can adversely affect our productivity, health, security, and quality of life. Noise generated by fans, vehicles, wind turbines, and propulsion systems are major contributors to this unwanted sound [2]. Acoustics is the incorporative science that accords with the study of mechanical waves in gases, liquids, and solids [3].

The noise source divides into two main categories: vibro-acoustics and aeroacoustics. Vibro-acoustics (noise-induced by structural) is the noise generated by the operation of equipment such as an engine that causes vibration in panels and surfaces. Vibro-acoustics noise also known as structure borne noise. On the other hand, the noise generated by air is called airborne or aeroacoustics noise. Aeroacoustics is a multidisciplinary engineering field that includes scientific subjects of the fluid mechanics and the classical acoustics. Aeroacoustics is also the study of noise generation by airflows, and the way in which aerodynamic systems can be designed to minimize noise [2]. In this field, the generation of sound by aerodynamic forces or flow perturbations is studied. In other words, aeroacoustics is a branch of acoustic science that studies the production of noise by turbulent flow, the noise generated by the impact of aerodynamic forces on surfaces, and/or the noise generated by periodic change in flow. Generally, Aerodynamic noise is often due to two completely different sources. The first one is related to the sound of surfaces movement in unsteady flow. The effect of displacement of a body in fluid and aerodynamic forces, cause pressure fluctuations that generate noise. 
The aerodynamic noise mainly comes from rotating systems (such as helicopter rotors, wind turbines, turbine engines and fans [4]. The second noise source or aeroacoustics mechanism is the result of turbulent flow, which exist in almost all engineering applications. Turbulence has a random nature and has a wide range of frequencies. The turbulent energy can be converted into acoustic energy. This energy conversion is often complete in areas of sharp edges, such as trailing edge of an aircraft wing. The other major source of sound is the jet stream, which combines shear layers to propagate sound. It is the act of combustion, which is the result of chemical interactions and the entrance of energy into the flow.

According to the above, the noise of turbulence flow usually exists. Fig 1 shows schematically the different sources of noise. For example, turbulent boundary layer flow, vortex shedding or shear layers noise [5].
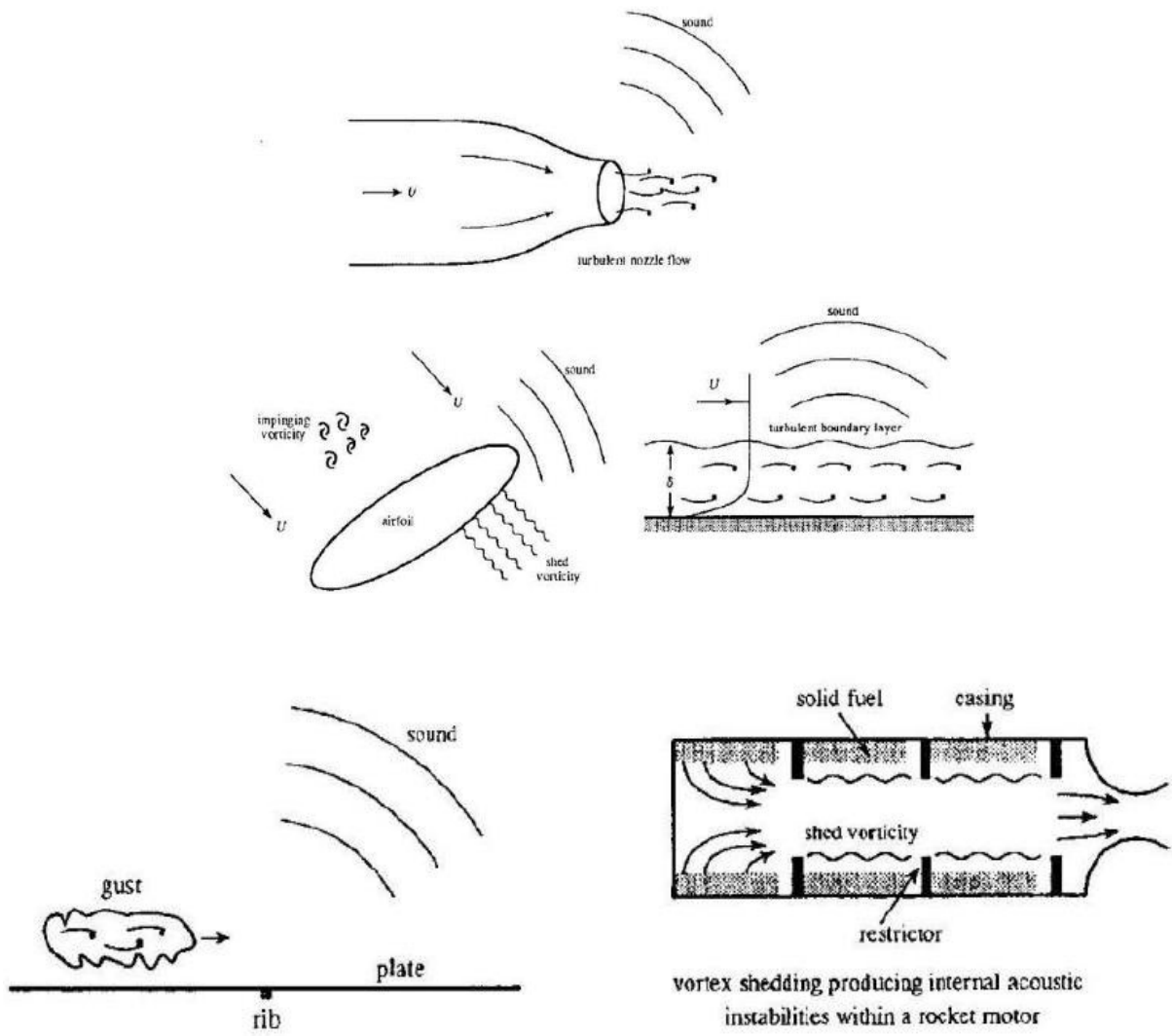

vortex shedding producing internal acoustic instabilities within a rocket motor

Fig 1. Noise source due to turbulence flow [5]

Until now, no integrated scientific theory of the emission of noise by aerodynamic flows has been established, most constructive aeroacoustics analysis relies on the so-called aeroacoustics analogy [6] which published by Sir James Lighthill in the 1950s [7]. In the first studies, experimental approach is used to estimate the propagation of sound waves. But nowadays, along with the increasing the computational power of computers, numerical simulations of noise generated by fluid flow, called Computational Aero-Acoustic (CAA), has been widely used by researchers [8-11]. 


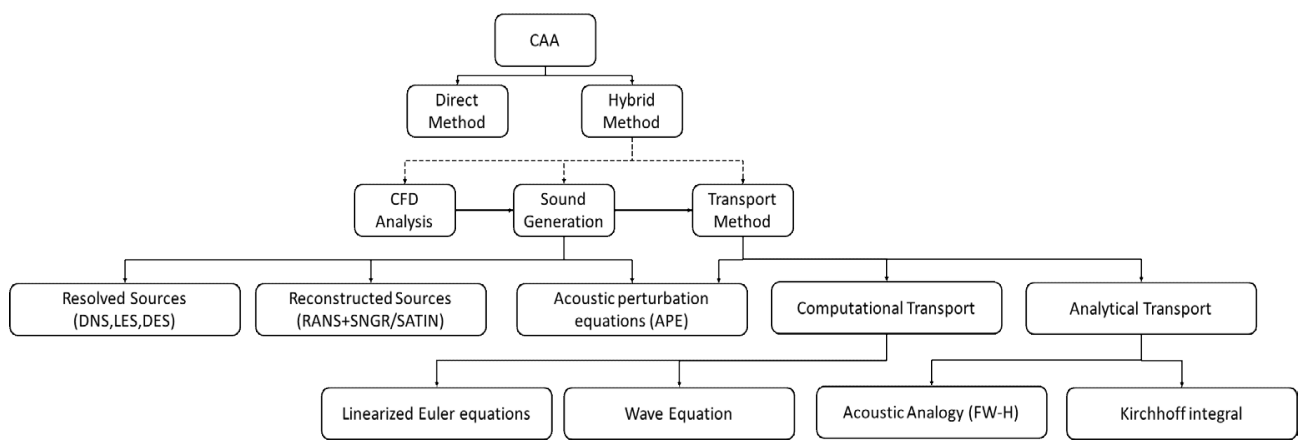

Fig 2. Noise Source Prediction approaches

In literature there are many definitions of CAA, but generally CAA is a branch of aeroacoustics that aims to analyze the emission of Aerodynamic noise through numerical methods .CAA use special numerical methods and related techniques, such as Lighthill acoustic analysis [6], Kirchhoff method [12], Ffowcs Williams-Hawkins equations [5], to capture acoustic phenomena. According to Fig 2, The CAA can be divided into two categories: direct and hybrid methods. In direct methods, noise generation and propagating phenomena, solve simultaneously. While hybrid methods use computational fluid dynamics (CFD) techniques and acoustic solver methods separately, In other words hybrid methods use CFD to solve flow field and numerical acoustic methods to find sound sources [13, 14]. One of the best hybrid CFD-CAA approaches consists of a large-eddy simulation (LES) to compute the unsteady turbulent flow field and a consequent CAA step, in which, e.g., the acoustic perturbation equations (APE) are solved [15]

It always has been difficult to calculate the noise generated by fluid flow due to the nonlinear governing equations. In addition to conducting experimental tests, there are many problems in calculating wind noise, such as separating background noise, as well as the model scale problem according to the Strouhal and Reynolds numbers. Nevertheless, todays many studies have been conducted by using the CFD and CAA tools in the field of the acoustic source mechanisms, noise prediction and aerodynamic noise reduction technologies, especially in aerospace engineering [4].

Aeroacoustics computing has a long history in fluid mechanics. Gutin (1948) published the first of these attempts in Russia [16]. Understanding the physical mechanism of aerodynamic noise generation is an important step to reduce or control noise emissions. As it mentioned above the basic principles of aerodynamic noise prediction in infinite flow using acoustic analogs first obtained by LightHill [6]; Curl [17] extended mentioned studies by considering the presence of solid boundaries in the flow field. Ffowcs Williams-Hawkins [5] proposed a method for considering the effects of moving surface on noise emission. The Ffowcs Williams-Hawkins formula derives from general form of LightHill acoustic analogy and it is able to calculate the noise produced by several acoustic sources. In this method, several surfaces can be considered as the noise source and receiver. Receivers can also be considered as stationary or moving at a constant speed [18]. This method assumes that there is no barrier between the noise sources and the receivers. Therefore, this method can be used to predict noise from external aerodynamic flows [5]. LightHill has also proved that aerodynamic noise sources can be categorized into several sets of monopole, dipole, and quadruple. 
Monopole sources represent the noise generated by fluid flow fluctuation due to the motion of the surfaces, dipole sources represent the noise generated due to the oscillations of force on the surface of the things, and quadruple sources represent the sound generated due to flow stresses fluctuations out of surfaces [5].

This paper outlines some applications for noise control in Aerospace, Automotive, and turbomachinery industries; also, critical noise source is reviewed, Highlights of aero noise control technique with an emphasis on cutting edge technologies is presented along with representative results and relevant conclusions. The main approach of authors in this review is the synergy of noise control technologies between different industries.

\section{AEROACOUSTICS IN AEROSPACE INDUSTRIES}

As noise problem became a major environmental challenge over the past years, the aerospace industries have paid special attention to Aeroacoustics for the design of aircraft. The worldwide air traffic is expected to double every 15 years and the annoyance to the population living in the vicinity of airports should at least remain the same [19]. It is obvious that more flights could be allowed if noiseless aircraft were used. Reducing aircraft noise is depend on many components such as wing or engine noise.

ICAO has adopted rules for Take-off and landing phases of aircraft noise that is mainly from the engine. According to the 2006 standard [20], aircraft should have a noise reduction of about $10 \mathrm{~dB}$ compared to the 2002 standard [21]. The details of how to measure sound are as shown in Fig 3.

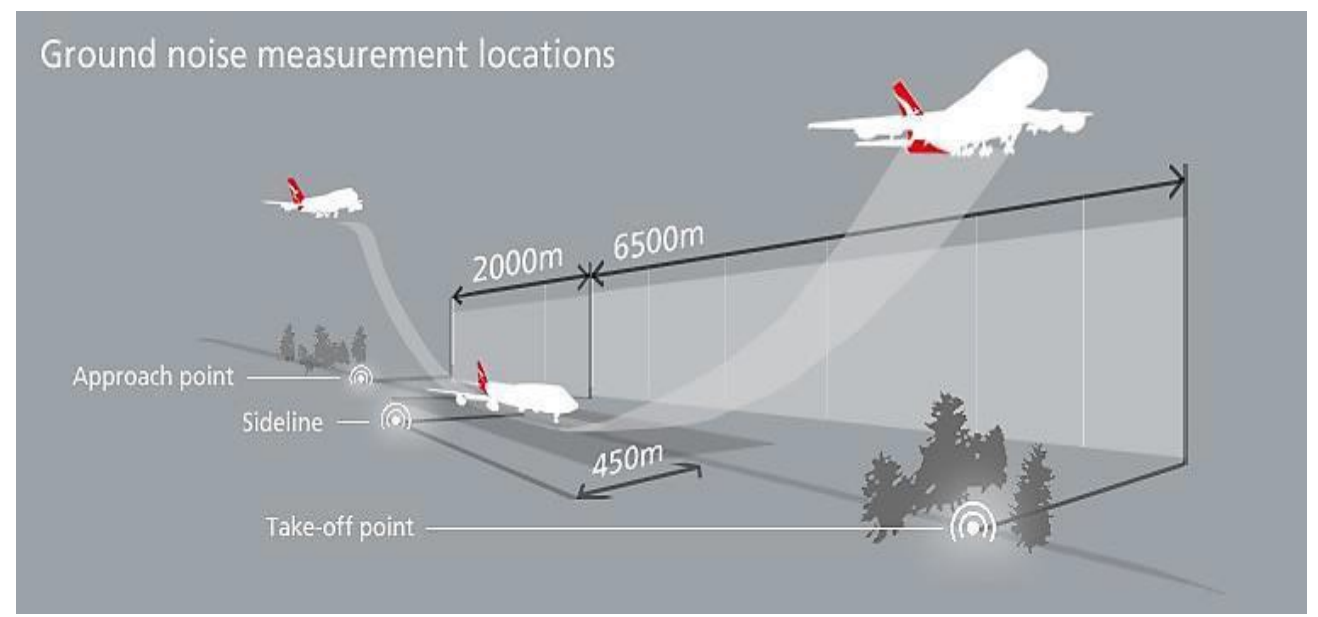

Fig 3. Noise measurement method [22]

The mentioned rules have made aircraft jet noise reduction to a noticeable issue in aeroacoustics researches. The main sources of noise in an aircraft are included aerodynamic noise, noise from aircraft systems, engines, and mechanical noise. Aircraft engines are the main sources of aircraft noise, especially when the aircraft is operating near the airport during takeoff and landing operating conditions [22].

Engine noise generally is due to the fan (including stators), outlet (the jet flow), the compressor, the combustion chamber, and the turbine; especially jet flow noise and fan noise are more important than other sources. It is generally desirable to reduce noise level without any effect on efficiency, which has been a challenge for recent years [22]. 
Clearly, the high-velocity jet flow ejecting out of the engine nozzle has inherent instability of the shear layer. This unstable flow resulting in vortices that eventually collapses into the turbulence zone where the jet noise source is generated. Various methods have proposed for reducing engine jet noise, without affecting other design parameters of the propulsion system. Some of these methods are the variable output nozzle using memory alloys [23-25], fan flow deflector [26-28] and the sinusoidal nozzles [29-31] as shown in Fig 4.

Since 1996, many numerical and experimental studies on the performance of the serrated nozzles are conducted [32].

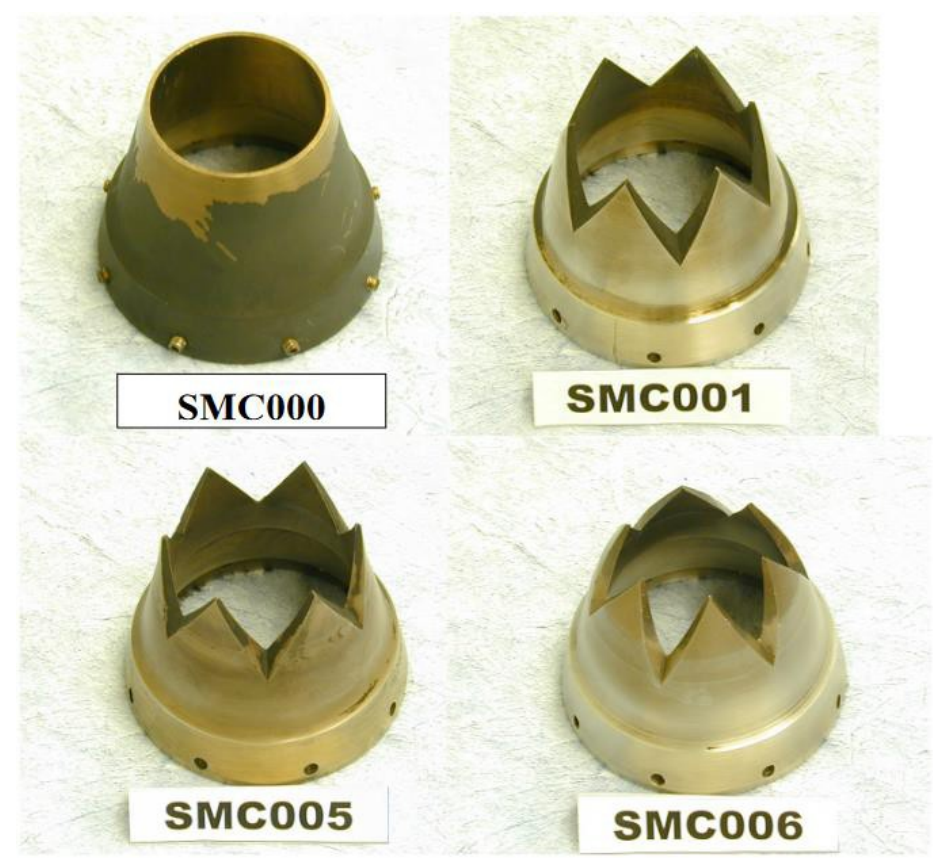

Fig 4. Some example of serrated Nozzles [32]

It can be concluded that the main source of noise in the nozzle is turbulent shear layer. Shear layer exists in downstream of flying objects, fluid jet and mixing layers and its main characteristic is formation and combination of vortices. These layers are very important because they dissipate energy and generate sound.

Due to the difference between the central flow and around flow at nozzle outlet, a shear layer is formed as shown in Fig 5. These turbulent structures have different length scales. Small vortices are created near the jet outlet and propagate in directions perpendicular to the jet outlet. These vortices grow as shear layers and gradually interact with small-scale turbulent structures. Large- scale turbulent structures also emit noise in the downstream direction [33].

In the aerospace field and in the case of airfoil, Chong et al. [34], by using trailing edge serration (Fig 6), achieved good results in noise reduction of an airfoil. Jaworski et al. [34] by using elastic material and Gruber et al. [35] by using combined trailing edge and leading-edge serrations (Fig 7) in a tandem airfoil attained acceptable outcomes. 


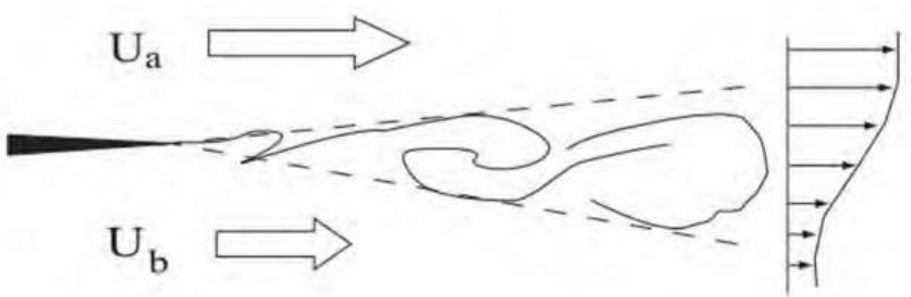

Fig 5. Free shear layer [33]

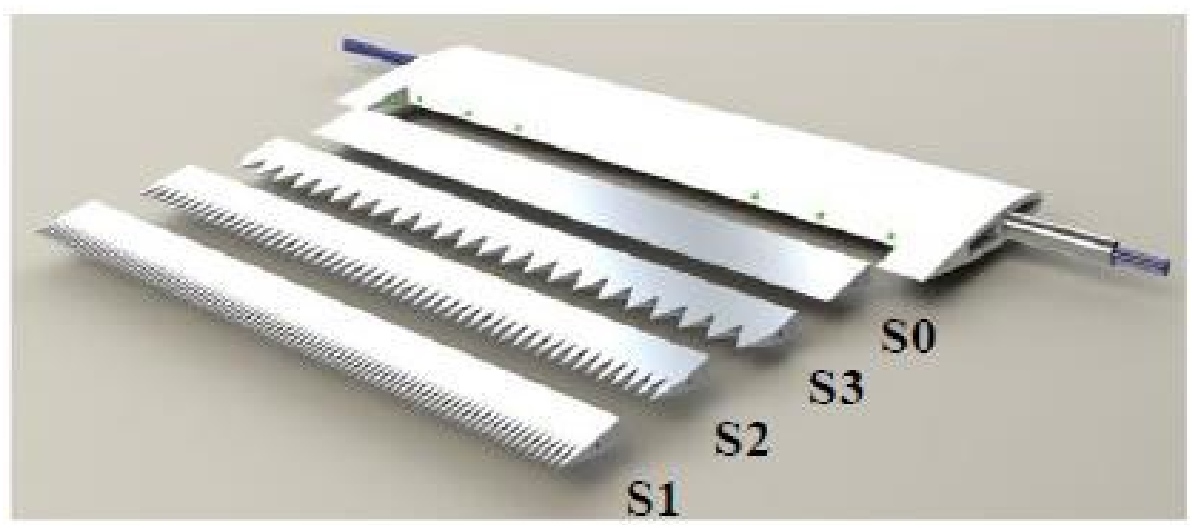

Fig 6. Test Cases which used in Chong et al. [34] work

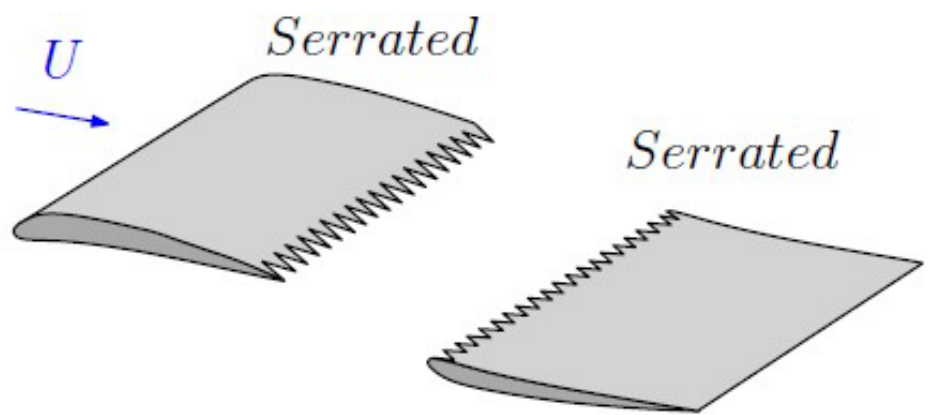

Fig 7. Test Cases which used in Gruber et al. [35] work

Strawn et al. [36] studied the aeroacoustics signal of a helicopter in forward and hover flight. They used the finite volume numerical method to solve the Euler and Kirchhoff equations for modeling the transmission of acoustic signal to the far-field flow. Their study showed that the acoustic oscillations obtained at high speeds in forward flight had good agreement with the experimental data. 
Recently Appelbaum et al. [37] performed a aeroacoustics simulation of a Gulfstream GIII aircraft by using PowerFlow software at Mach number of 0.23 and Reynolds number equals to 10.5 million (Fig 8). They investigated the noise in different flight phases by considering

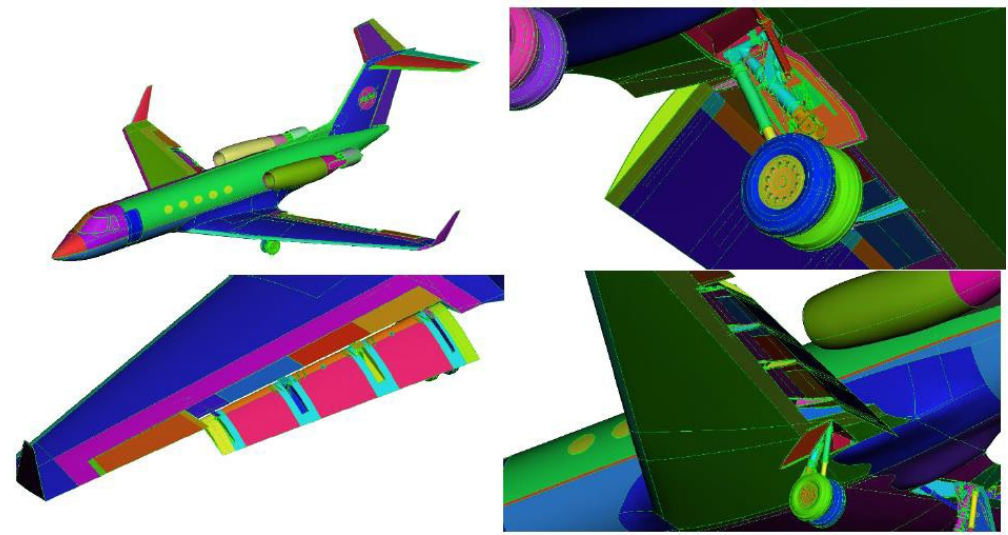

Fig 8. Airplane model used by Appelbaum et al. [37]

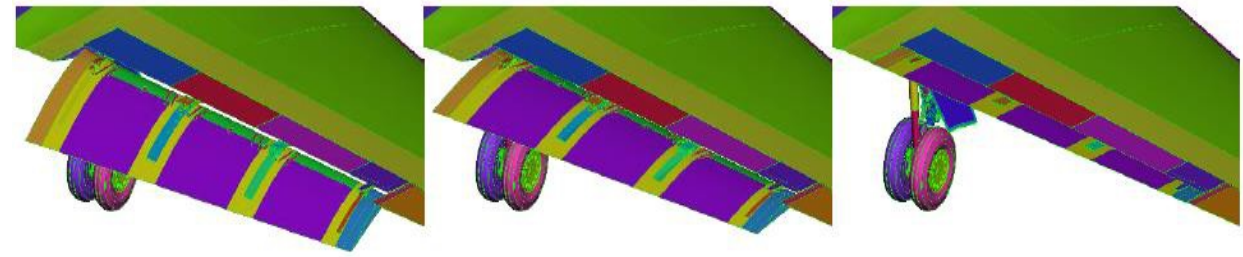

Fig 9. Flap change effects modeled by Appelbaum et al. [37]

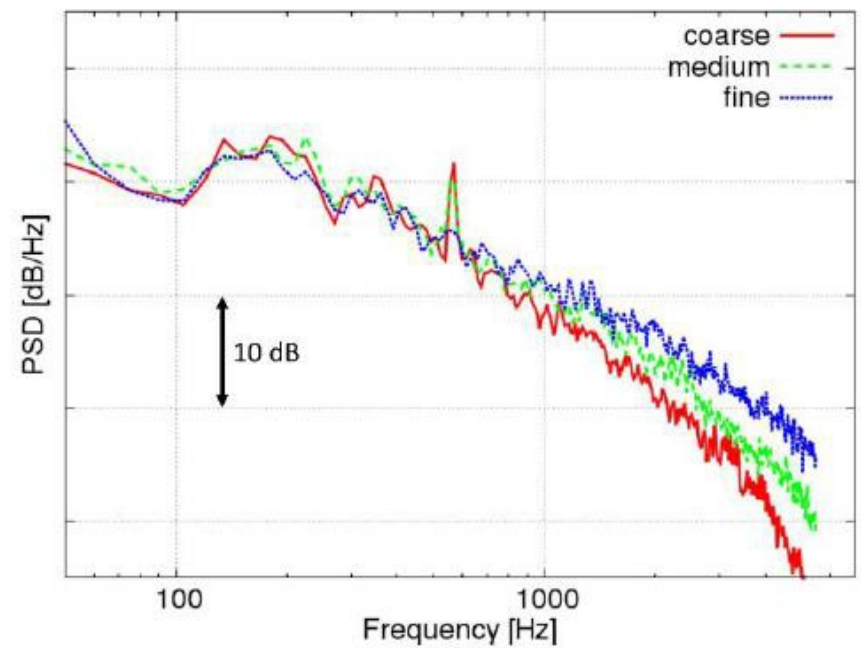

Fig 10. The effect of mesh size on Power spectral density studied by Appelbaum et al. [37] 
the effect of flap deflection (Fig 9), closing or opening landing gear. They used Ffowcs Williams-Hawkins method for simulating acoustic, and the Boltzmann method for simulating flow field.

As shown in Fig 10, in the mentioned simulation, the effects of three different mesh sizes are evaluated. This study showed that the grid size absolutely could affect the results, especially at high frequencies.

By investigating the effects of the flap angle on the noise level (According to Fig 11), their result showed, when comparing the effect of the Main Landing Gear (MLG) only, an increase of $20 \mathrm{~dB}$ is seen across the entire spectrum. Comparing the effect of flap deflection, an increase over the entire frequency range becomes visible. Their results also showed that, the drop-off remains at a constant level because of the strong noise sources at the flap edges.

Arcondoulis et al. [38] presented a numerical and experimental study of noise in a cylinder coated with a porous media (Fig 12). Study of the flow through the cylinder is a common test case in aerodynamic. The flow studied in Reynolds numbers from 0.08 to 0.02 million. Three types of porous surfaces with different porosity levels including polyurethane, metal foam and structured evaluated and their results showed that using the porous surface, specially polyurethane foam is effective in reducing the tonal noise caused by vortex shedding (Fig 13) [38].

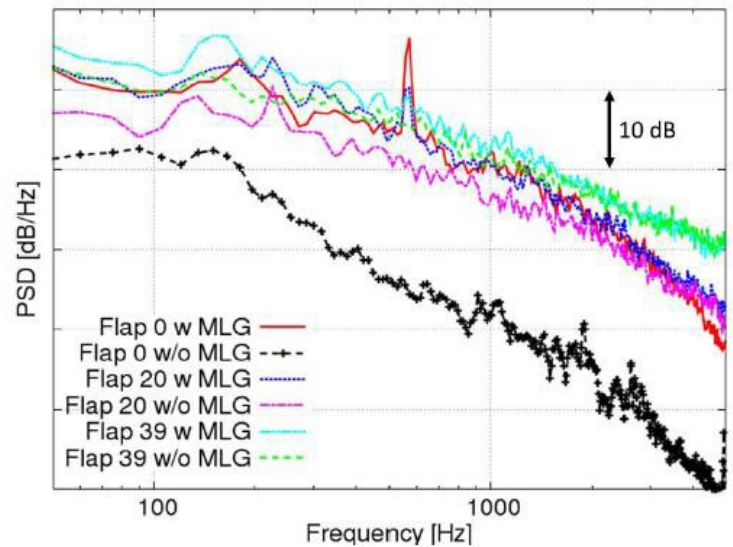

Fig 11. The effect of flap angle on Power spectral density studied by Appelbaum et al. [37]
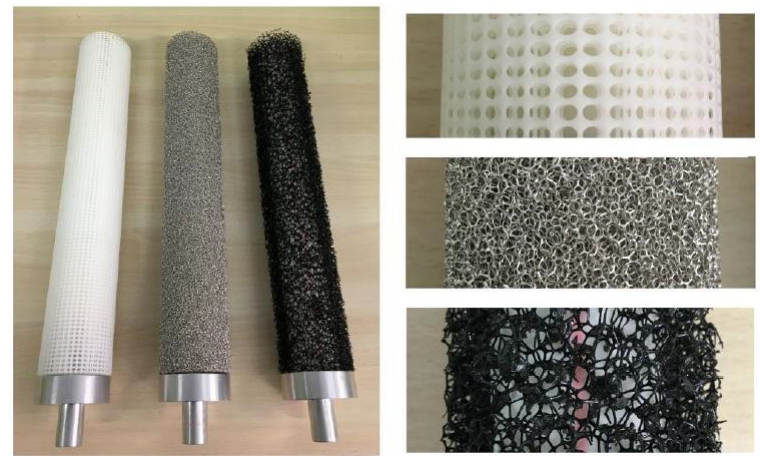

Fig 12. Different porous cylinder studied by Arcondoulis et al. [38] 
Biedermann et al. [39] investigated the effect of using serrated trailing edge (as shown in Fig 14) on aerodynamic noise of an airfoil. The past studies have showed serrated trailing edge is very effective in stall angle delay, but aeroacoustics consideration with this control method discussed in this paper. Their model was Airfoil NACA65 (12)-10 and the noise measurement method was beam forming as shown in Fig 15.

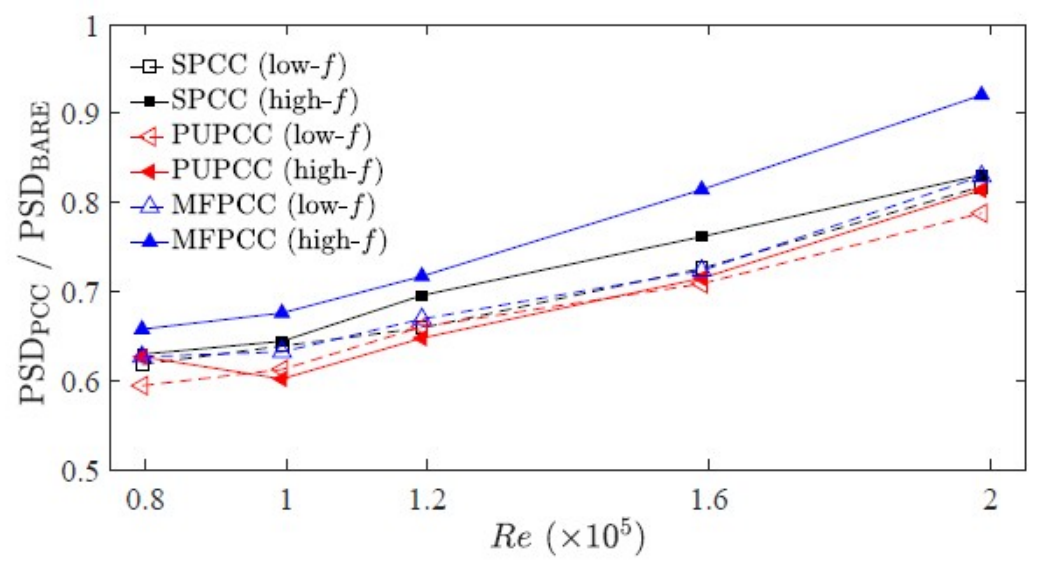

Fig 13. Comparison between three types of foam in different Reynolds studied by Arcondoulis et al. [3]
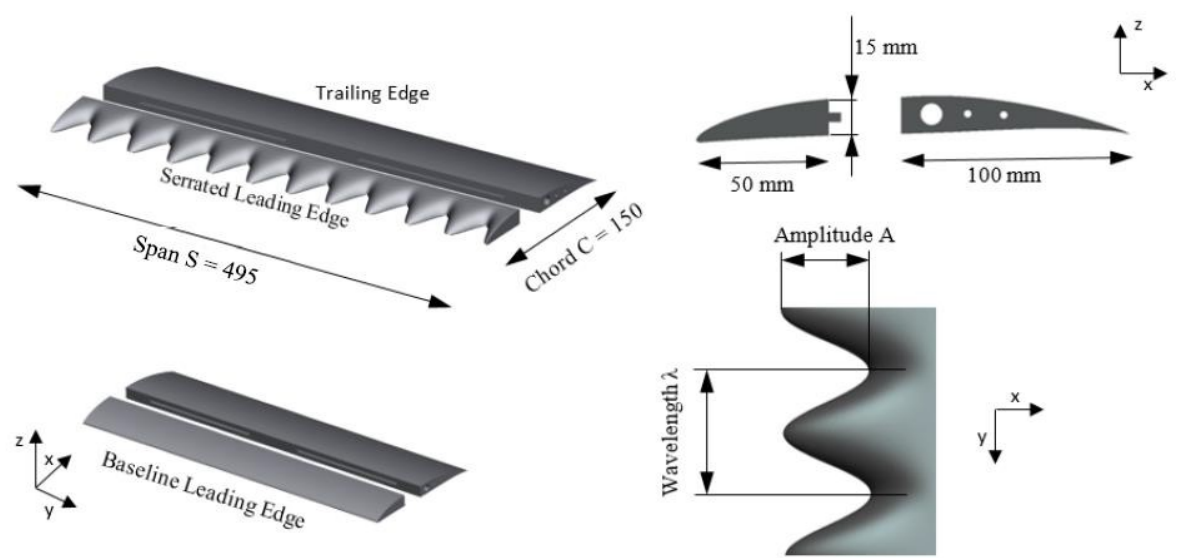

Fig 14. Serrated airfoil studied by Biedermann et al. [39]

As shown in Fig 16, the results showed that the noise can be reduced by this method about $12 \mathrm{~dB}$. The results also disclosed a strong dependency of inlet turbulence intensity on the noise level and high turbulence of the inlet flow improves the aeroacoustics performance. The effect of the angle of attack (as shown in Fig 17) also investigated in this paper [39].

Chirico et al. [40] investigated the aeroacoustics performance of the Fokker-50 engine's propeller (Fig 18). As shown in Fig 19, various propeller and hub designs evaluated [40]. Their result of the sound pressure levels for modified designs relative to the baseline propeller 
showed that the off-loaded tip blade has tones at lower frequencies, was lower than the Baseline design, with an appreciable noise level reduction up to at least the fourth tone. Staggered and Unequally Spaced designs had tones also at multiples of BPF1s/2.

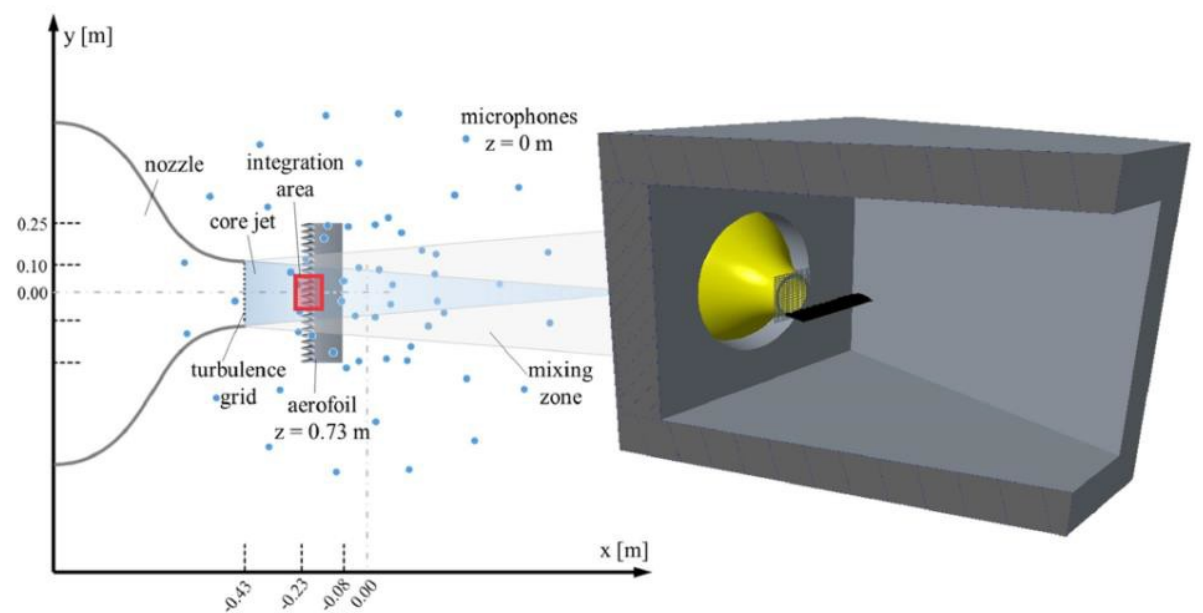

Fig 15. The location of microphones in the Biedermann et al. [39] test stand

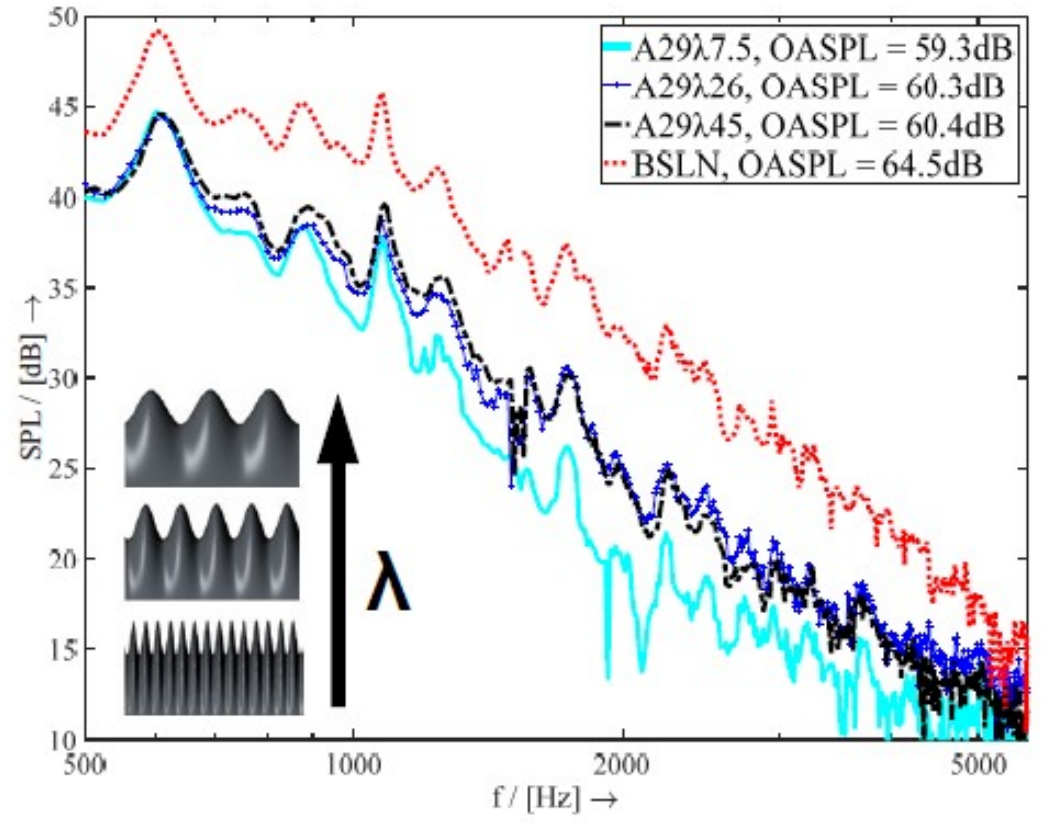

Fig 16. The effect of serrated shape studied by Biedermann et al. [39]

In a work recently published by $\mathrm{Li}$ et al. [41] the three-element airfoil 30p30N in the high lift condition experimentally investigated (Fig 20). According to their results as shown in Fig 21 the tonal noise decreased with increasing angle of attack. 

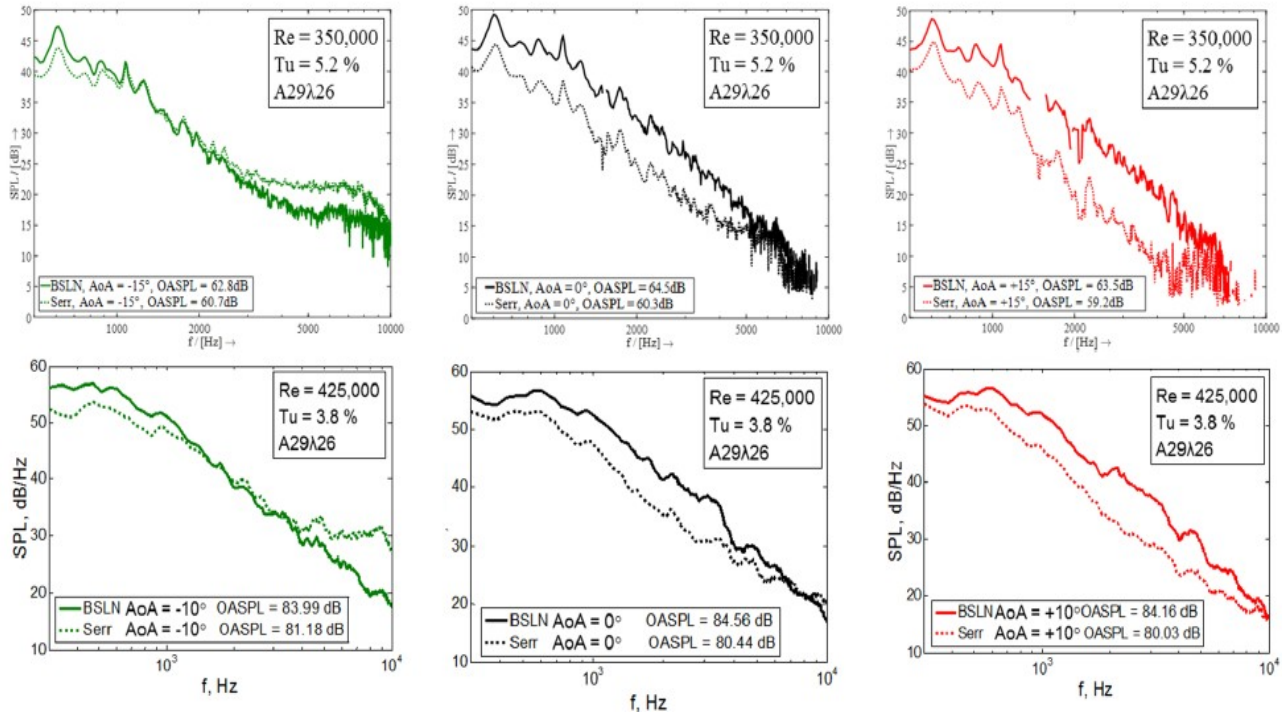

Fig 17. The effect of angle of attack studied by Biedermann et al. [39]

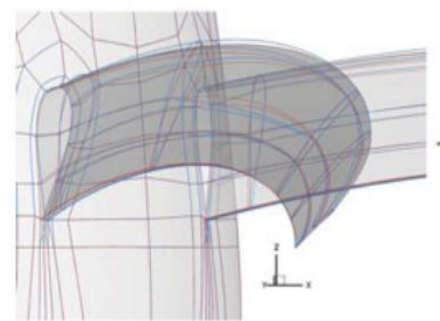

(a) Offloaded Tip blade (light blue).

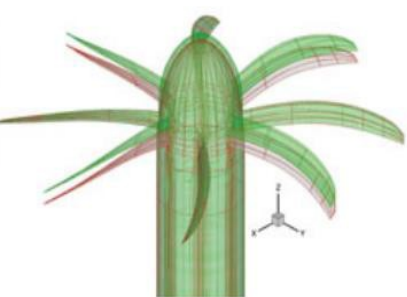

(b) Staggered hub (green).

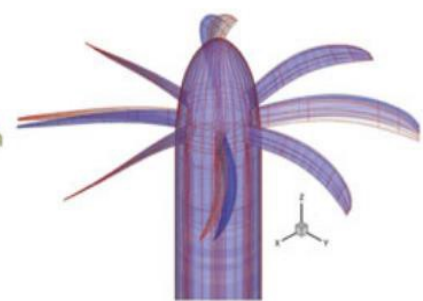

(c) Unequally-spaced hub (blue).

Fig 18. Modified propeller geometries vs Baseline design, which investigated by Chirico et al. [40]
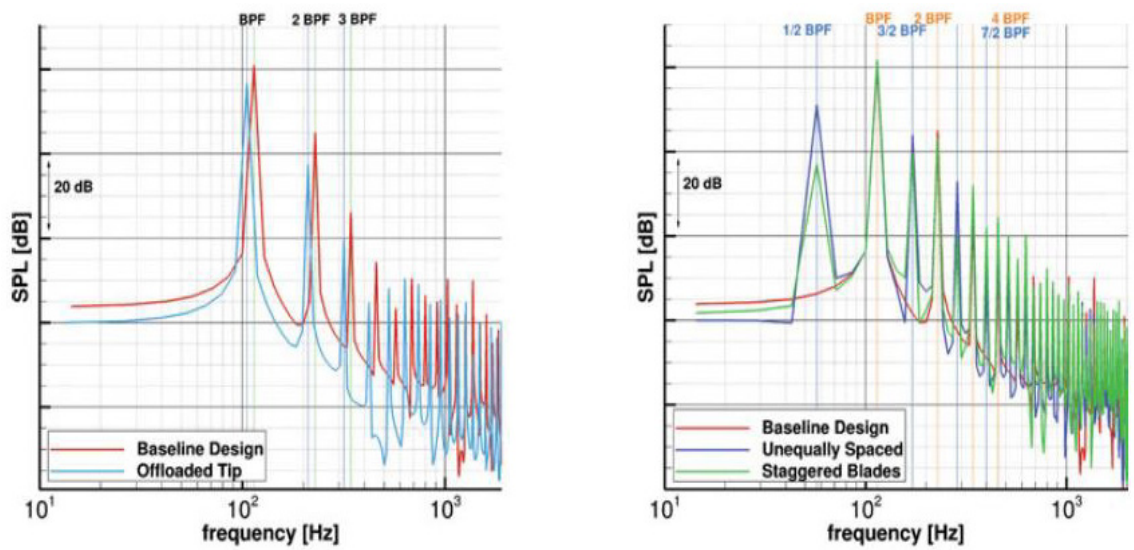

Fig 19. Sound pressure level for different propeller design, which studied by Chirico et al. [40] 


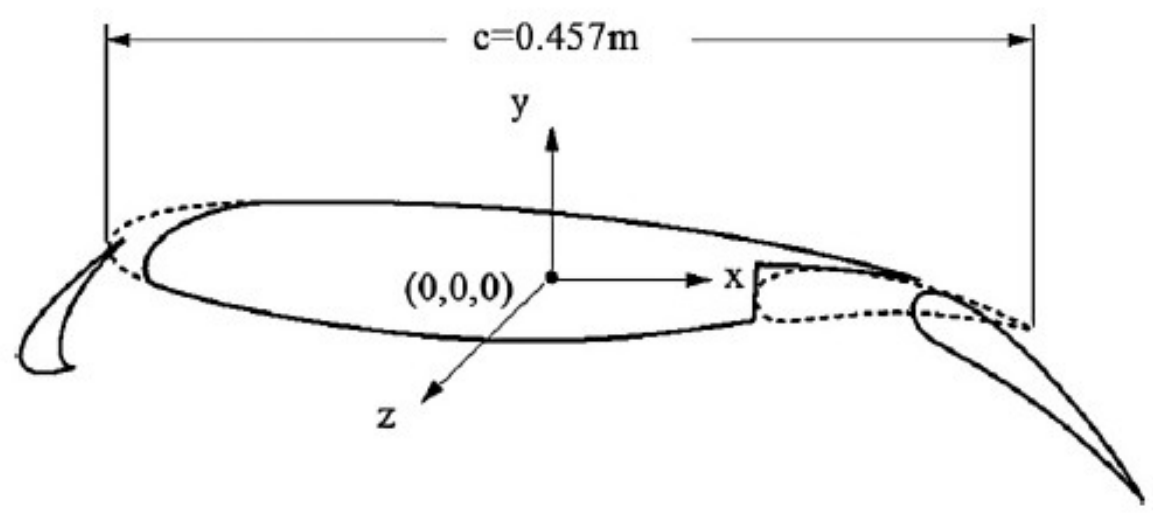

Fig 20. 3 Element airfoil acoustically studied by Li et al. [41]

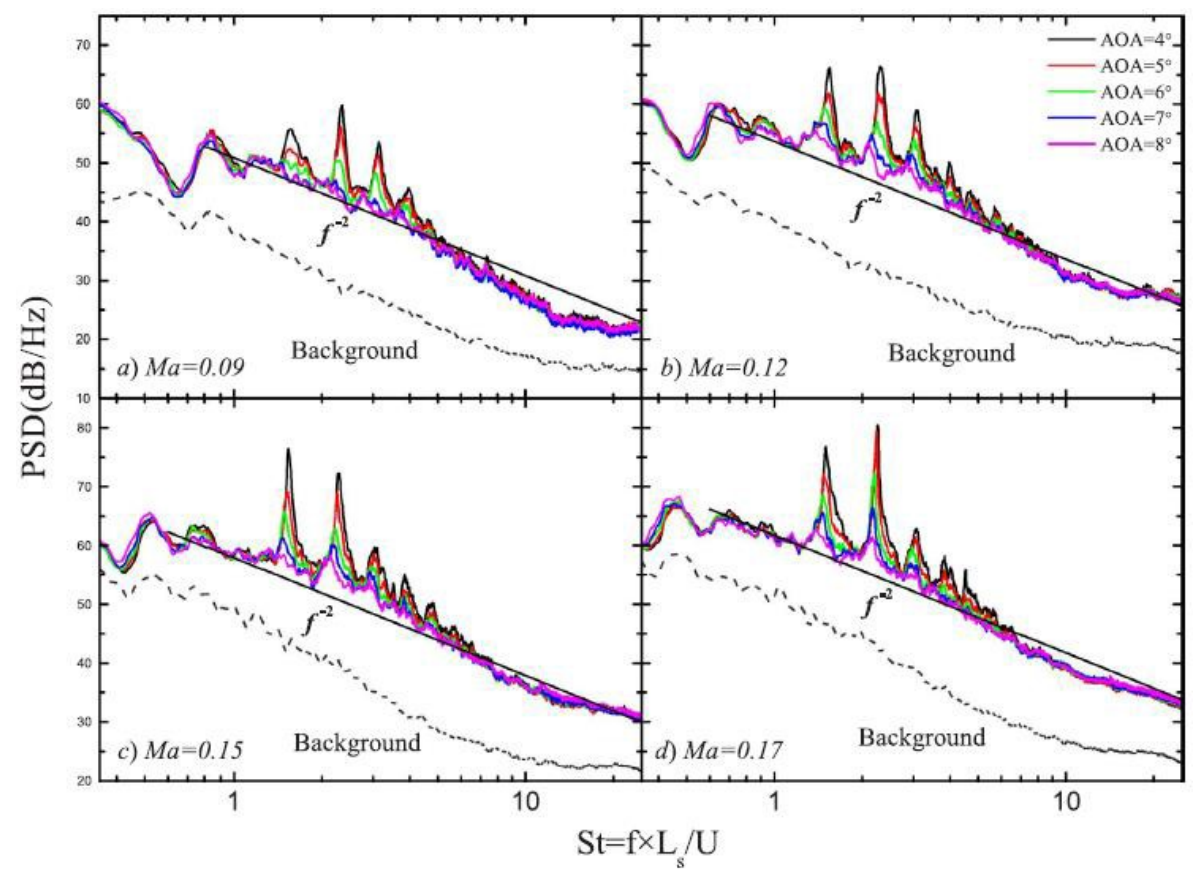

Fig 21. The effect of angle of attack on noise level studied by Li et al. [41]

Takaishi et al. [42] reduced the landing gear generated noise by designing an innovative device (Fig 22). In their device a small wind deflector on one side installed upstream of the gear well to deflect shear flow away from the cavity opening and reduce low -frequency noise generated when the flow hits the rear edge of the cavity. Porous material with open cells used to the inner surface of the gear to suppress local flow-induced pressure fluctuations. Holes in the landing gear wheel sealed with aluminum tape to suppress the aerodynamic sound generated from air passing through them. Finally, their device decreased aircraft noise about $4.5 \mathrm{~dB}$ as shown in Fig 23 


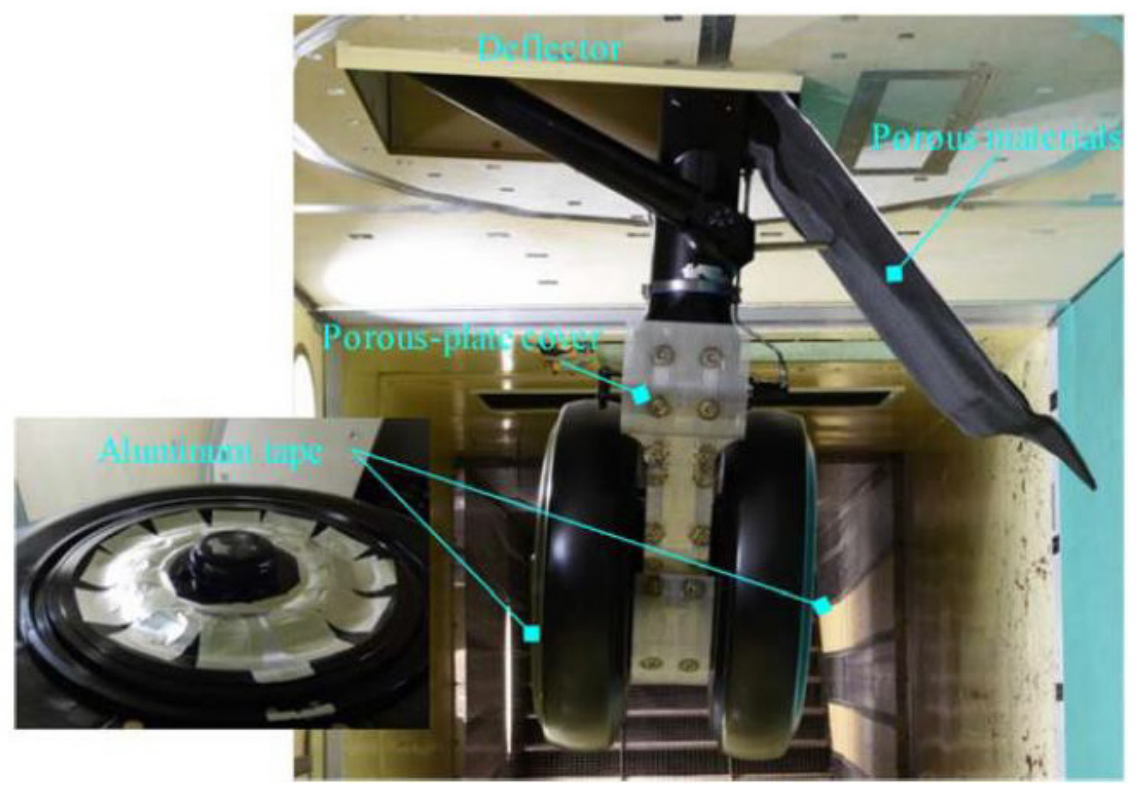

Fig 22. Innovative designed device for reducing noise generation studied by Takaishi et al. [42]

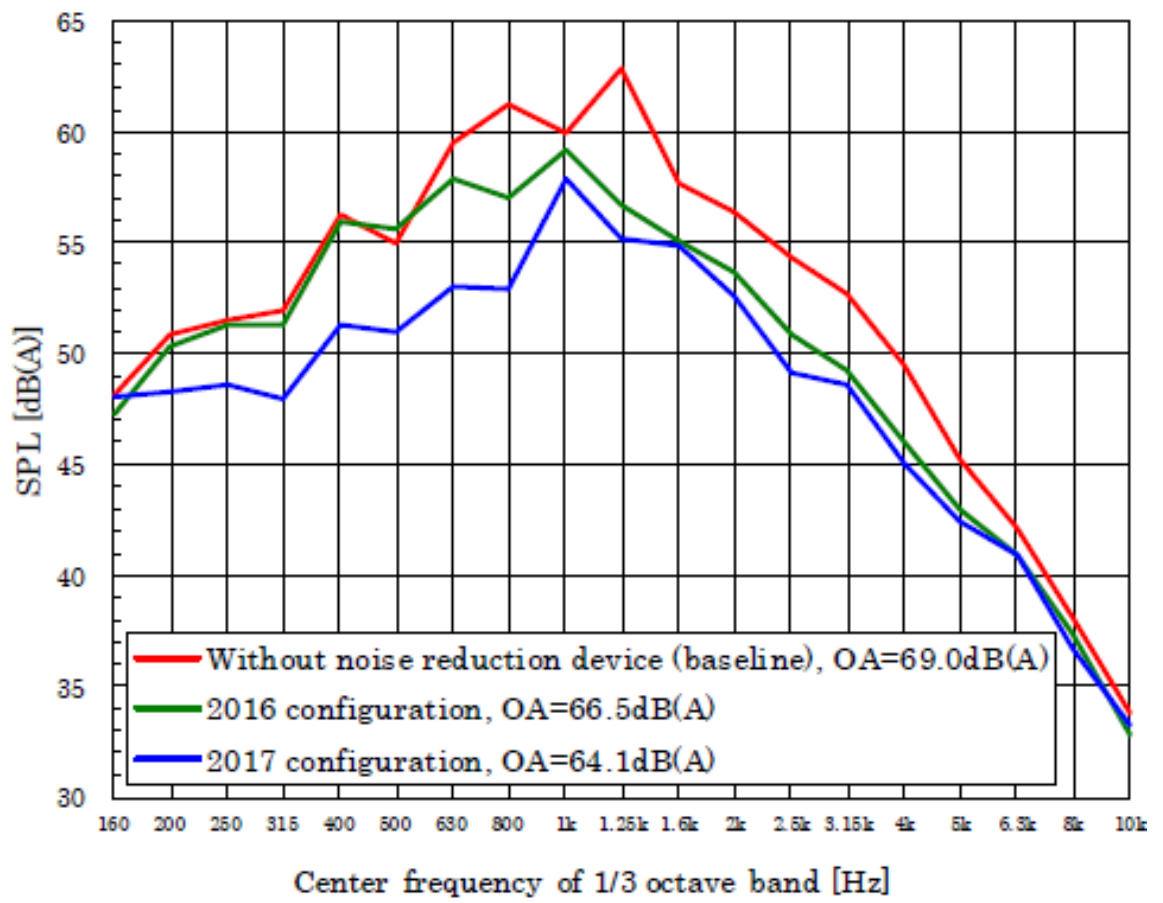

Fig 23. Sound pressure level comparison studied by Takaishi et al. [42] 


\section{AEROACOUSTICS IN TURBOMACHINERY INDUSTRY}

Turbomachinery, in mechanical engineering, describes machines that transfer energy between a rotor and a fluid, including both turbines and compressors [43]. Especially for turbines, recently and due to the extreme demands for energy, there has a rapid development of wind turbines [44]. The major issue of the wind turbines is the emitted noise, which affects the nearby residents; so it is a need to estimate aerodynamic noise emitted by wind turbines and to find noise reducing technique in order to increase public approval of wind energy [45]. Sound emitted from a turbomachinery could be due to mechanical or aerodynamic sources. Mechanical noises are generated by various mechanical components and equipment, including motor and gearbox, and aerodynamic noises are caused by blades that mainly interact with the turbulent flows [46].

Numerous experimental and numerical studies have been carried out to study the aeroacoustics phenomenon in turbomachinery. Kuntz et al. [47] showed that reducing the size of the blades reduces sound generation but it also decreased the machine efficiency too, so they increased the rotational speed to compensate it.

A study on the aeroacoustics of six-blade impeller (Fig 24) by Lieser et al. [48] showed that by decreasing the impeller size at a constant rotational speed, the sound emission is significantly reduced. Fehse et al. [49] studied noise generation mechanisms of low-frequency centrifugal fan. Jones et al. [50] used numerical simulation to design a rotorcraft airfoil with minimum noise generation. The results showed that unvented shaped airfoils had good aerodynamic and aeroacoustics performance.

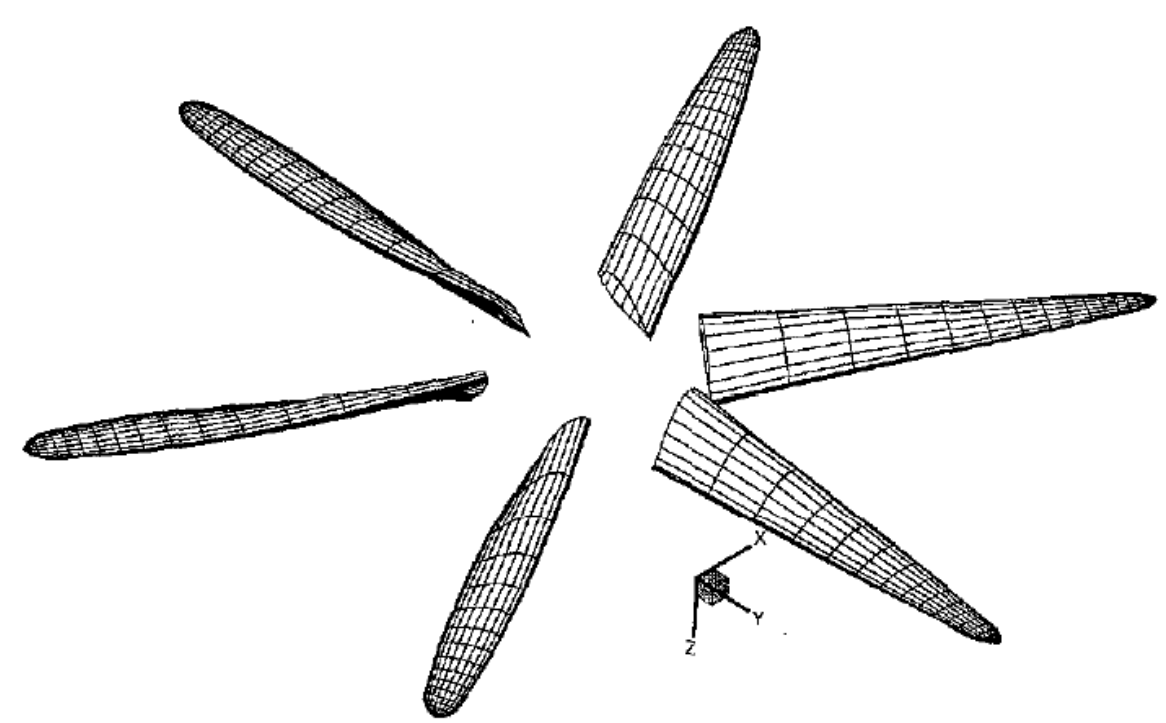

Fig 24. 6 Blade-impeller shape which used by lieser et al. [48]

Chapman [51] investigated analytically the acoustic phenomenon of high velocity flows with computational aeroacoustics codes. The source of the noise in this work was convected gust striking the leading edge of a wing or fan blade at arbitrary subsonic Mach number; the stream wise shape of the gust was top-hat, Gaussian, or sinusoidal, and the cross-stream shape 
was top-hat, Gaussian, or uniform. The results of chapman are useful for benchmark testing of computational aeroacoustics codes.
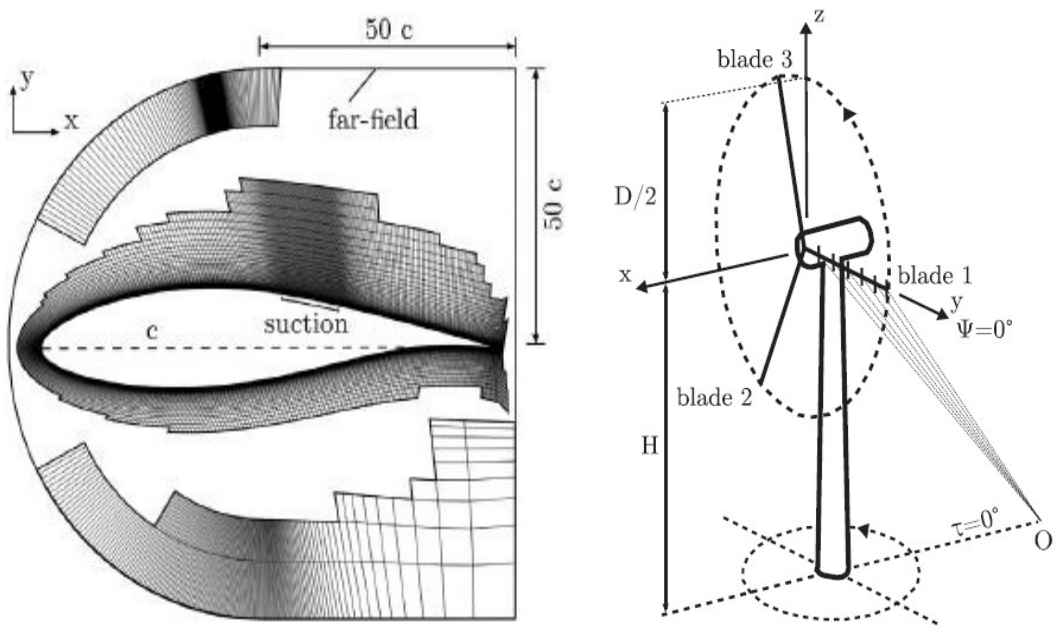

Fig 25. The location of suction studied by Arnold et al. [44]

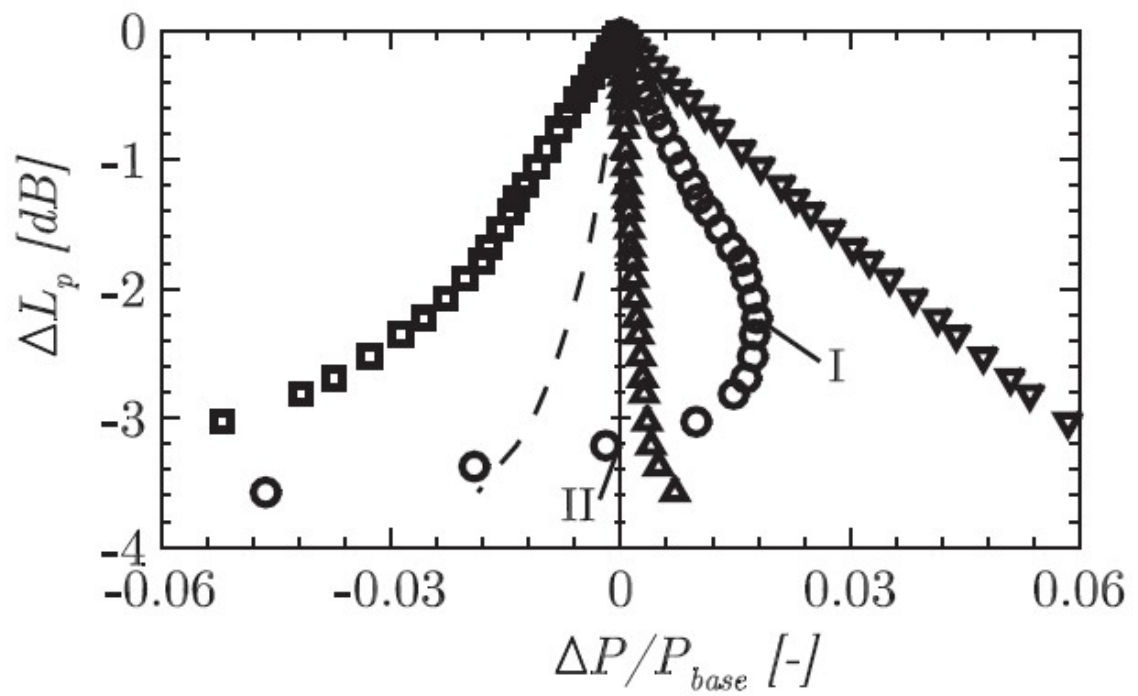

Fig 26. The effect of suction on emitted noise studied by Arnold et al. [44]

Kim et al. [52] investigated the sound generated at different wind speed in different wind direction and its effects on aerodynamic performance of wind turbine; their results showed that aerodynamic performance increases with decreasing noise emission level.

Mohamed [53, 54] investigated the effect of velocity, blade shape, and blade effects on the sound emitted by Darius H-shaped wind turbines. 
Recently, Arnold et al. [44] investigated the effects of suction on trailing edge noise and power of a wind turbine (Fig 25 and Fig 26). The aim of this research work was to achieve a trade- off between turbine noise reduction and efficiency improvement [44].

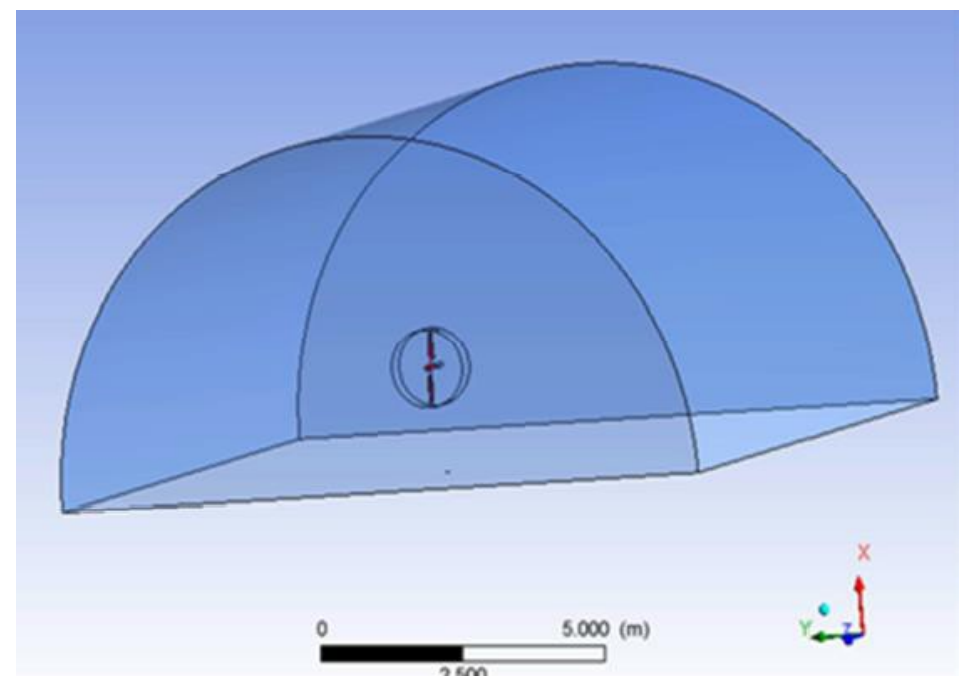

Fig 27. Computational Domain which used by Maizi et al. [55] for modeling horizontal axis wind turbine
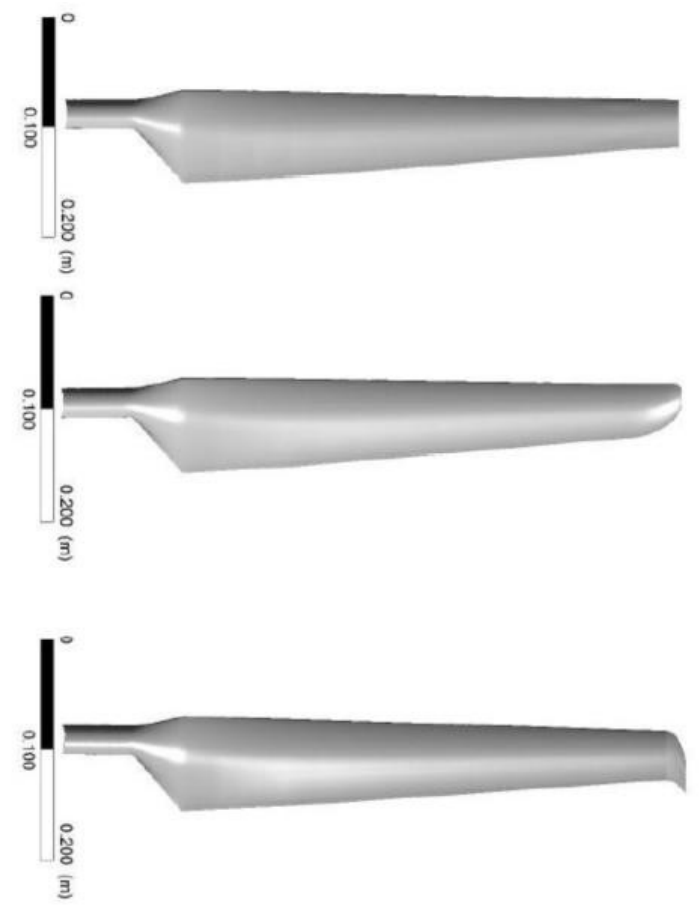

Fig 28. Different shape of propeller examined by Maizi et al. [55] 
Maizi et al. [55] investigated the noise generated in a horizontal axis wind turbine affected by blade tip shape (Fig 27). In this work, different tip shapes examined as shown in Fig 28.

The turbulence model in this work was DES and the Ffowcs Williams-Hawkins method used to model the aeroacoustics phenomena. The results according to Fig 29 showed that the use of unvented tip shape is very effective in reducing noise level [55].

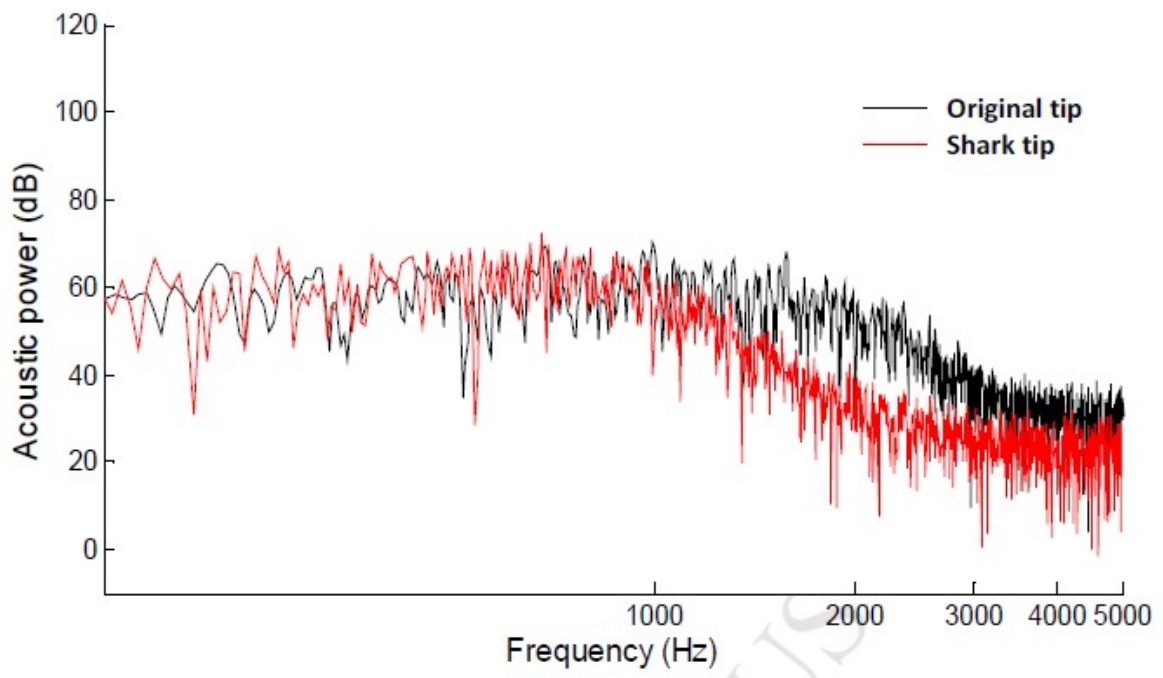

Fig 29. Comparison between acoustic power generated form blade with shark tip and original tip studied by Maizi et al. [55]

\section{AEROACOUSTICS IN AUTOMOBILE INDUSTRY}

Since noise, vibration and harshness (NVH) are now important in passenger vehicles, automotive Industries are interested in levels of NVH as a component of the development of comfortable vehicles. In particular, aerodynamic sound is the principal component of interior vehicle noise at speeds above $100 \mathrm{kph}$ [56]. Various design techniques have been developed to reduce the aerodynamic noises induced by high-speed driving. Many efforts have been expended on the Sound quality evaluation and improvement of vehicle aero noise in the past few decades [57-60]. The main noise source in the vehicle as shown in Fig 30 include the engine, power transmission, tires, aerodynamic pressure distribution around the body, exhaust gases and body vibrations. In Automotive engineering, vibro-acoustics noise is predominant between 0 and $250 \mathrm{~Hz}$ at low speeds (below $100 \mathrm{~km} / \mathrm{h}$ ) and Aeroacoustics noise is predominant above $500 \mathrm{~Hz}$ and at high speeds (above $1 \mathrm{~km} / \mathrm{h}$ ) [61].

The parts of the vehicle that cause turbulence in the flow and thus generate aerodynamic noise includes:

- Side mirrors

- A-Pillar

- Bumper edges 
- Front windshield

- Wiper

- A-Pillar gap

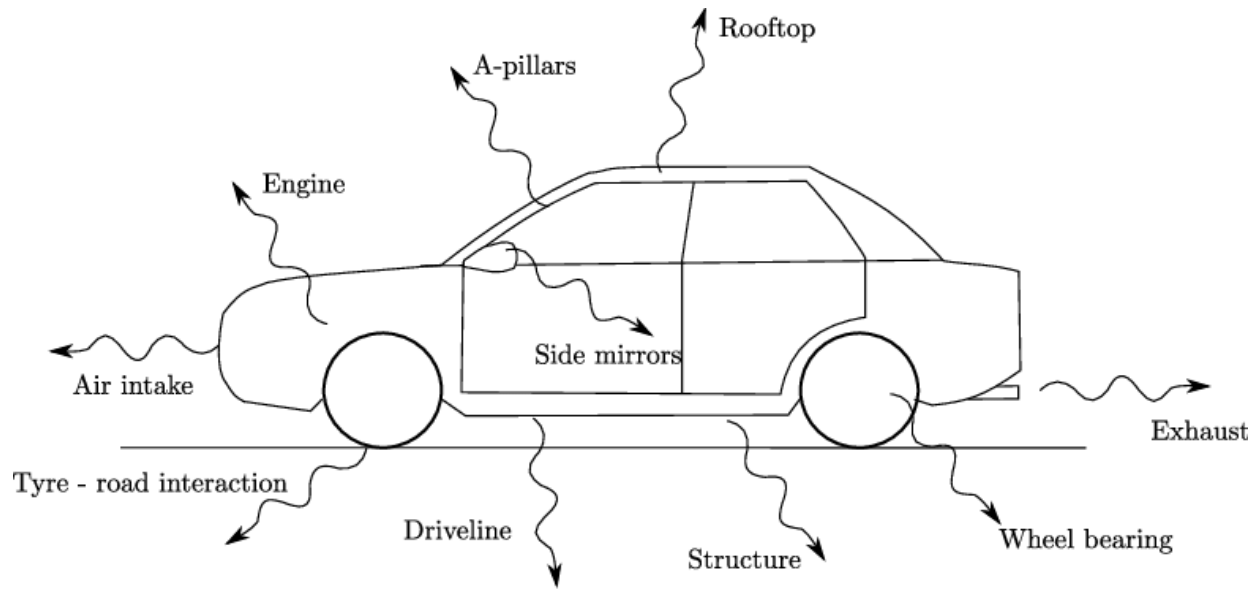

Fig 30. Noise sources of a vehicle [61]

In detail, the main aerodynamic noise sources in a vehicle could be categorized as follows:

- Leak noise as shown in Fig 31 is generally caused by air flow between the inside and outside of the vehicle. There is a pressure difference between the inside and outside of the moving vehicle, which causes the air to flow between them. For example, if the leak is considered to be a round gap with $4 \mathrm{~mm}$ diameter on a sheet with $4 \mathrm{~mm}$ thickness, this leak will produce a sound with a 0.6 Strouhal number or approximately $4400 \mathrm{~Hz}$. In general, the noise generated by a leak is the dominant noise in the acoustics. The most probable locations for this type of noise are the areas around the door and glass seals [61].

- Even if there is no leak on the body of car, the existence of cavity on the exterior of the car can generate noise. This noise is much higher in areas where the flow rate is high (such as A-Pillar). Probable points for this type of noise are the gaps in the A-Pillar, the gaps in the exterior of the mirror and the glass seams [61]. The mechanism for generating this type of noise is defined in two ways (Fig 32): The separation of the flow at the trailing edge of the cavity which can cause vortex shedding and sound production, and the second one, the separation of flow and vortex formation at leading edge of the cavity and collision of these vortices with cavity trailing edge which could be a source of noise (such as helicopter blades noise in very large scale)

- Even if the flow is not separated from the vehicle surface, due to highly turbulent flow and pressure fluctuations on the vehicle surface, Wind Rush Noise is always exist and unavoidable (Fig 33). Therefore, even in the absence of other types of noise, this noise could not be zero [61]. 


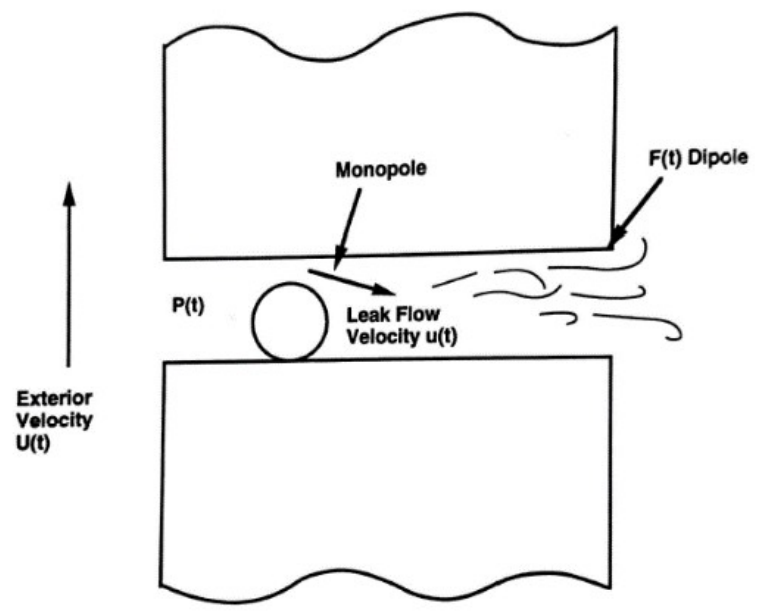

Fig 31. The flow structure in leak noise generation [61]

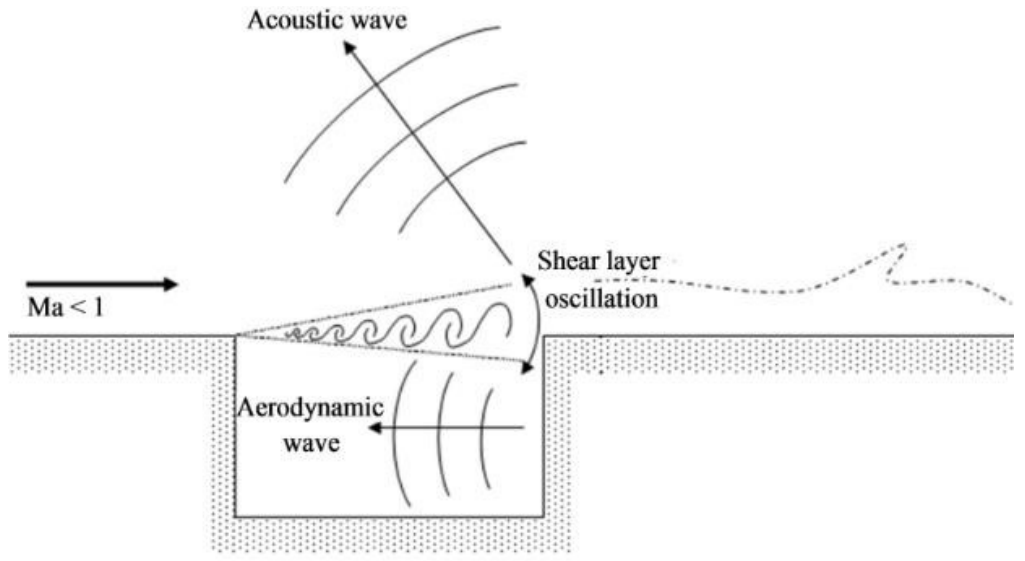

Fig 32. The flow structure in cavity noise generation [61]

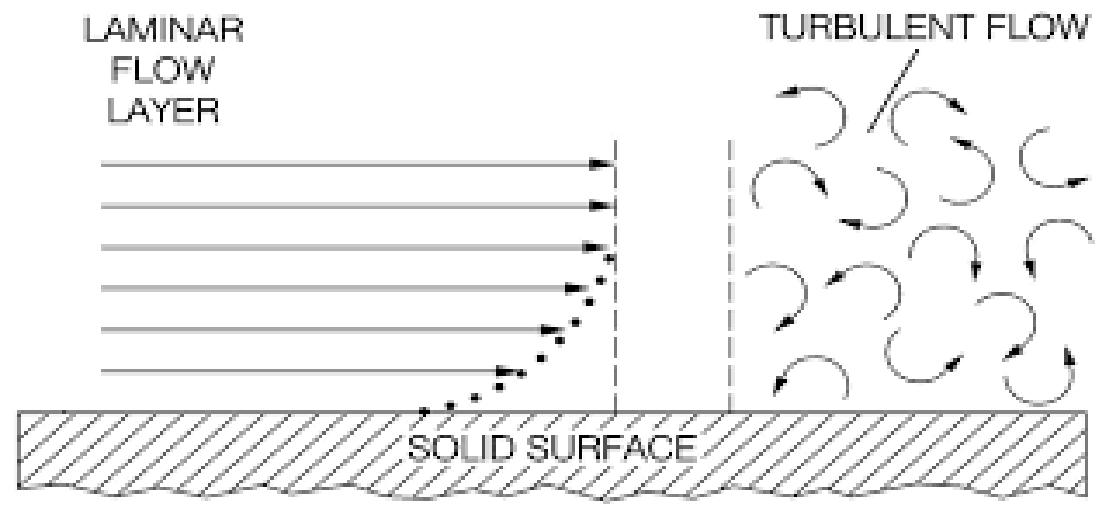

Fig 33. The flow structure in wind rush noise generation [61] 
One of the parts that has a major impact on the drag coefficient and noise of the car is the geometrical shape of the vehicle behind section. This noise generation and drag increase are due to the separation of the flow at this area and the formation of large-scale wakes. So, automakers todays have focused on design improvement of this area. It should be again mentioned that wake formation is one of the main reasons of noise. These wakes are formed in parts such as A-Pillar, B-Pillar, rear glass, automotive tail and side mirrors. The study of aerodynamic noise in these areas has been popular for researchers [62-65]. For example, Tsai et al. [66] performed an aeroacoustics analysis on the rear spoiler (Fig 34) and investigated its role on aerodynamic and aeroacoustics of the whole vehicle. They found that installing a spoiler with a proper angle of attack could reduce the aerodynamic lift coefficient. In addition, the spoiler also reduces the noise in the tail of the car. Shojaee-Fard et al. [67] investigated the flow around the A-pillar and the related aerodynamic noise. Their results showed that the corner radius (fillet) of the A-pillar and the windshield had a significant effect on the noise generation of them.

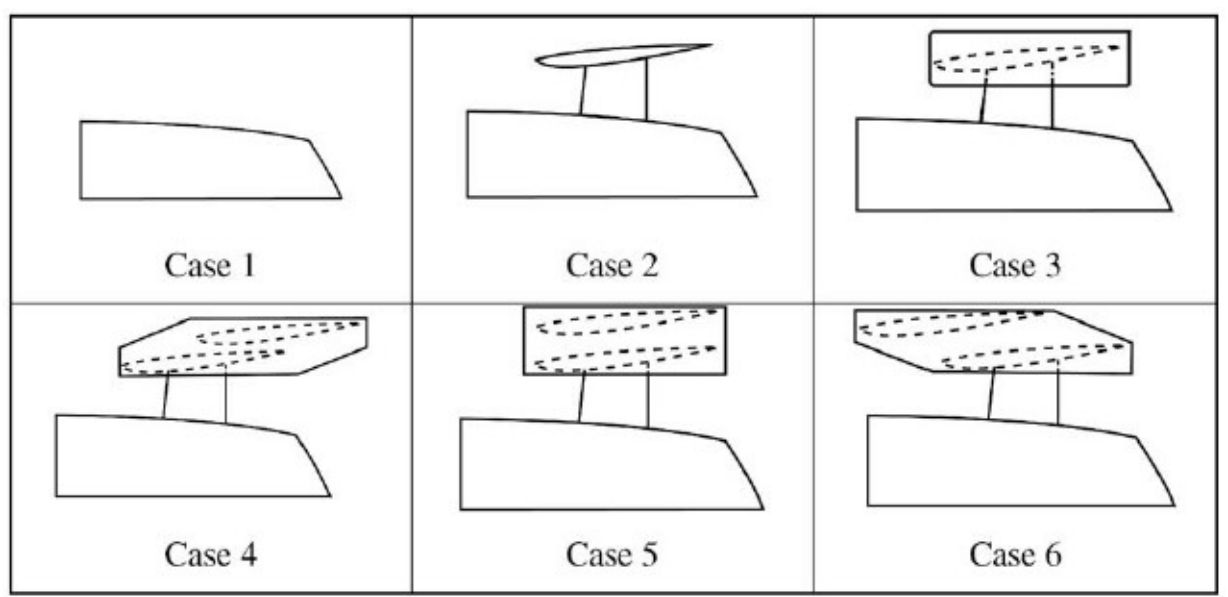

Fig 34 Various spoiler which used in Tsai et al. [66] work

The engine cooling fan is also recognized as one of the main noise source in automotive engineering. Wu et al. [68] developed a semi-empirical formula for the axial flow fan noise spectrum in a free flow field. The main assumption in the extracted formula was that the fan noise is caused by the fluctuation of forces from the fluid around the blades during fan operation. This interaction of structure and fluid creates a range of sound spectrum at different frequencies that is important in the blade passage frequency (BPF) and its harmonics. Since the noise generated by fan rotation originates from both turbulent flow and structural sources, one way of reducing the noise is to control the fluid flow through the fan. Vad et al. [69] proposed a method for redesigning the fan to reduce aerodynamic noise and improve fan efficiency. Thomas Kalmar et al. [70] tested an industrial axial fan and measured the noise emitted upstream of the fan by Phased Array Microphone (PAM) method. Further details about this method are available in the work of Benedek et al. [71]. On the other hand, the beam-forming method creates a pattern of sound intensity due to the phase difference between the input signals to the microphone [72]; The results of this method is used to identify noise sources. Investigation of axial Turbomachinery with beam forming method distinguishes rotor 
noise from other noise sources [72]. Classical noise identification methods had the capability of detecting static noise sources [72], while the Rotating Source Identifier (ROSI) algorithm is also able to estimate the location of rotating sources [73].

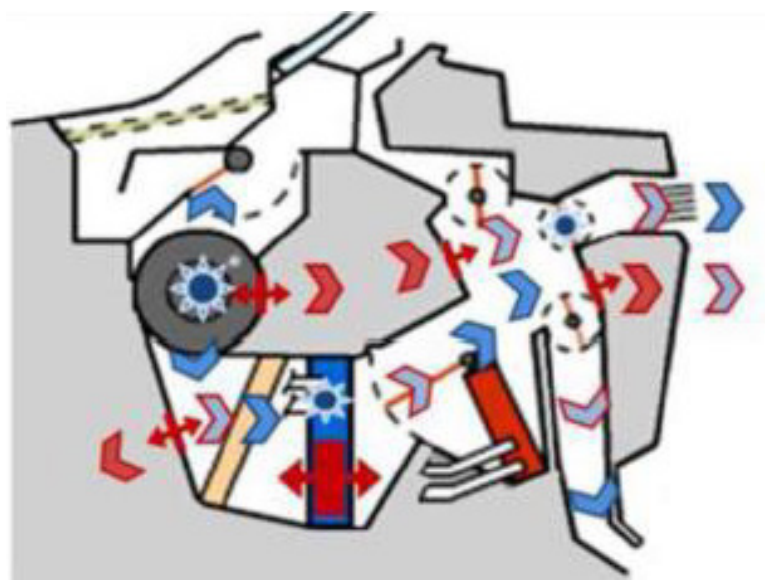

\section{Aeroacoustic source}

Vibration source

D Vibrations

D Aeroacoustic noise

D Vibration noise

Fig 35. Automotive HVAC Noise Source studied by Bennouna et al. [75]

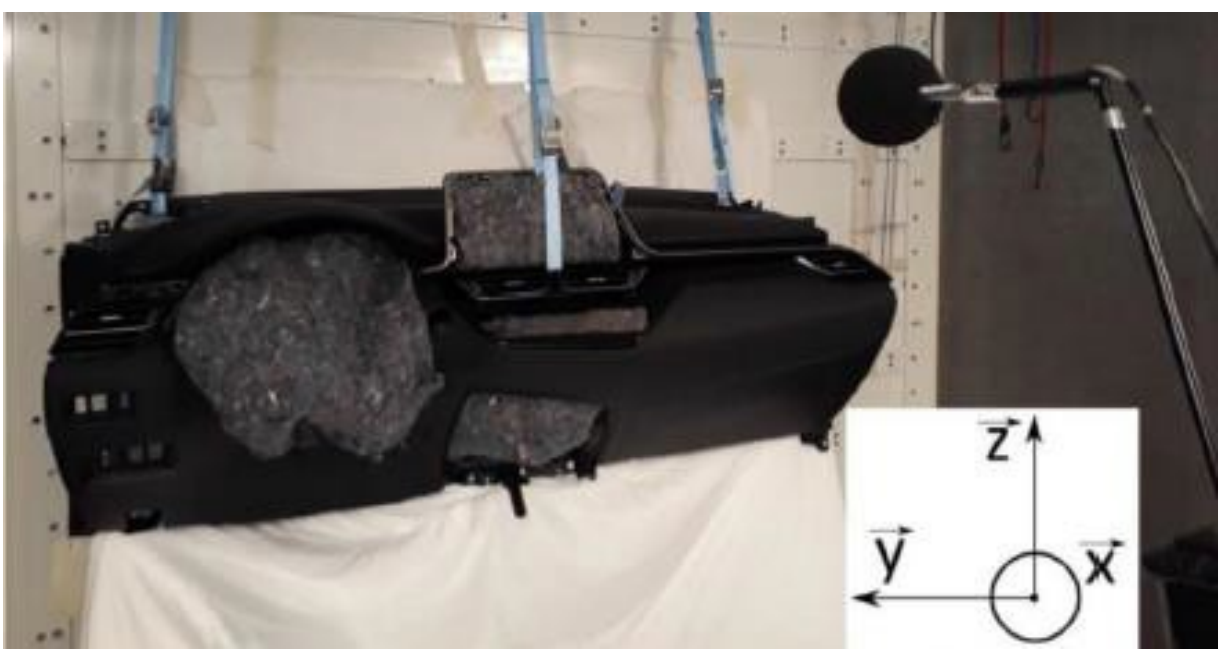

Fig 36. The usage of noise absorber which investigated by Bennouna et al. [75]

Fan noise could be tonal or broadband. Broadband noise sources can be classified as follow [74]:

- Turbulent flow

- Noise caused by the interaction of turbulent flow with the surface of the blades

- trailing edge noise

- Vortex shedding noise

- Noise caused by flow separation 
Recently Bennouna et al. experimentally and numerically investigated HVAC of the vehicle in order to reduce aerodynamic noise and improve the comfort of the occupants. In this work, after identifying noise generating points (as shown in Fig 35), they used different composition of sound absorbers as a passive method (Fig 36) to achieve the best noise reduction level (as shown in Fig 37).
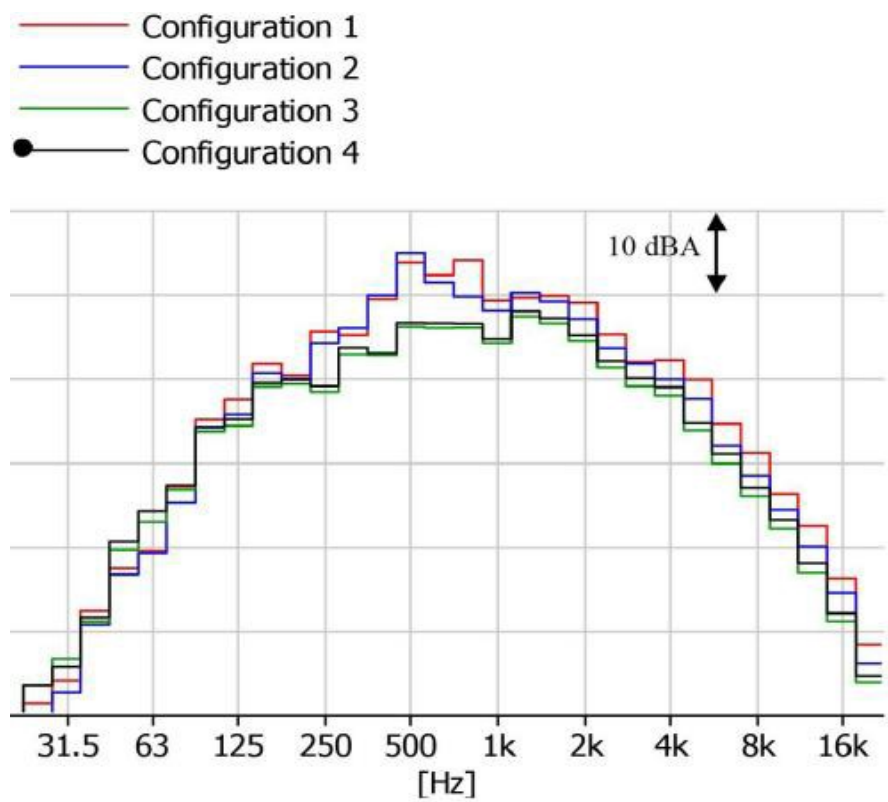

Fig 37. Effect of different configuration on noise generation studied by Bennouna et al. [75]

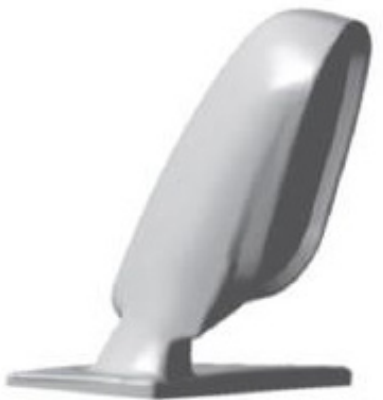

(a) The original model

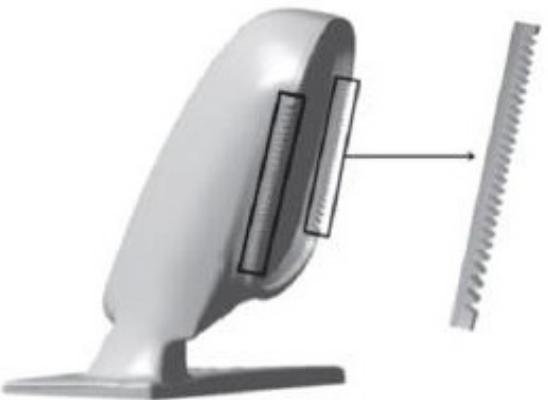

(b) The modified model

Fig 38. computational model studied by Chen et al. [76

Recently, Chen et al. [76] numerically and experimentally studied the effect of side mirror shape on aerodynamic noise. The turbulence model used in the numerical solution was LES model. Two types of mirrors with and without indentation studied (Fig 38). The numerical results of this work have showed in Fig 39 and Fig 40. The results showed that with the use of indentation mirrors, the pressure oscillations and vortices in the back of the mirror reduced and therefore noise level reduced (Fig 41). 


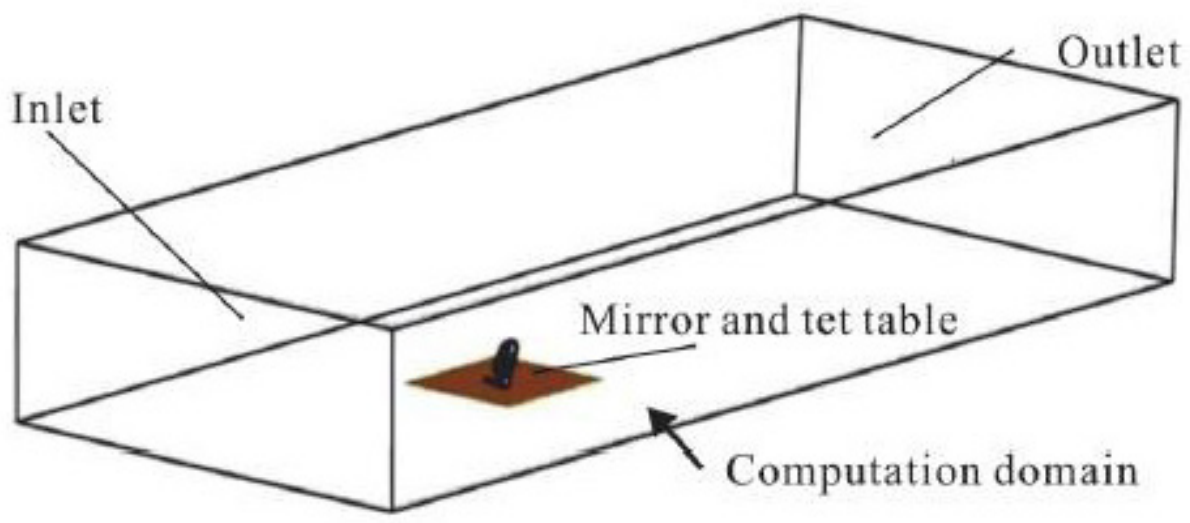

Fig 39. The Computational Domain which used by Chen et al. [76]
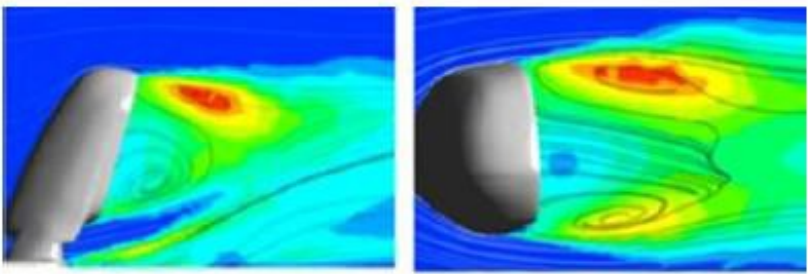

(a) The original model
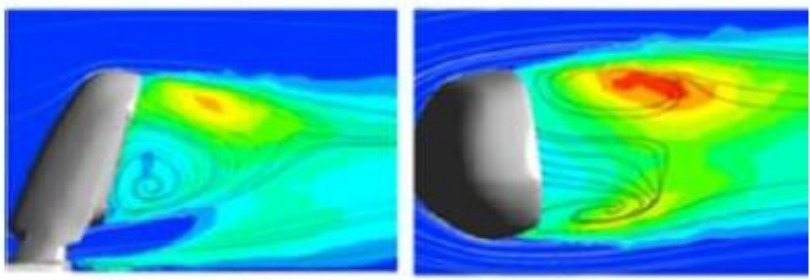

(b) The modified model

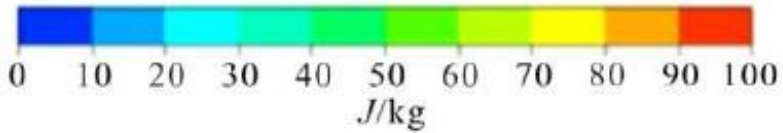

Fig 40. Turbulence kinetic energy around side mirror studied by Chen et al. [76]
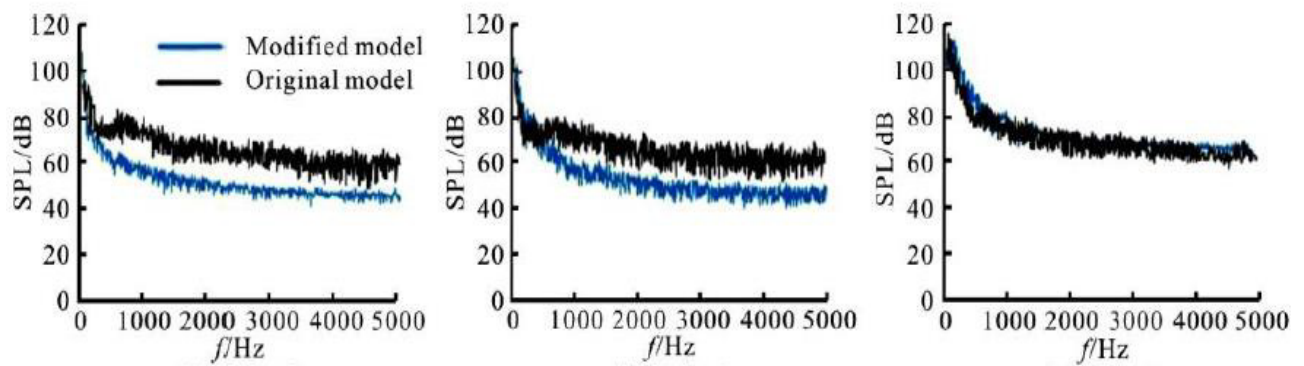

Fig 41. Comparison between noise level at different location of modified and original side mirror studied by Chen et al. [76] 


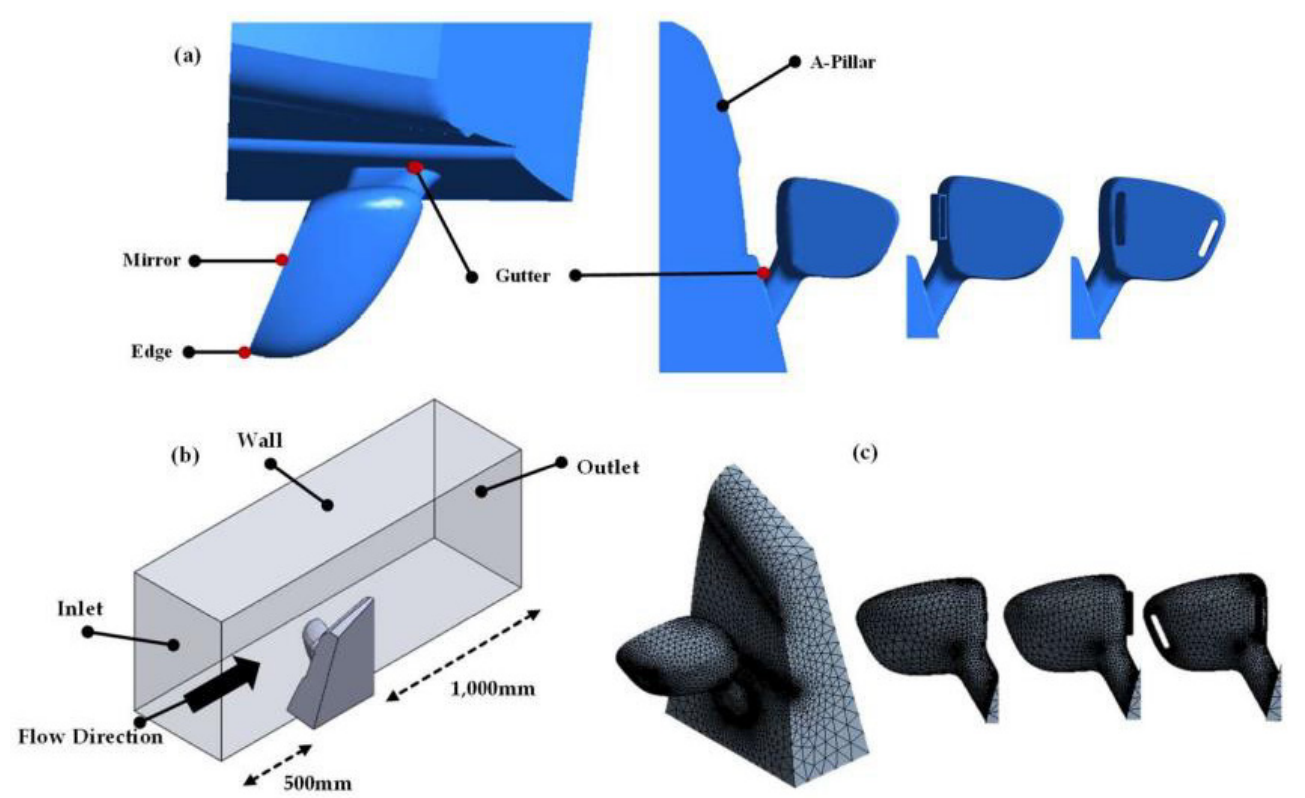

Fig 42. Computational Model studied by Yong-Ju Chu et al. [77]

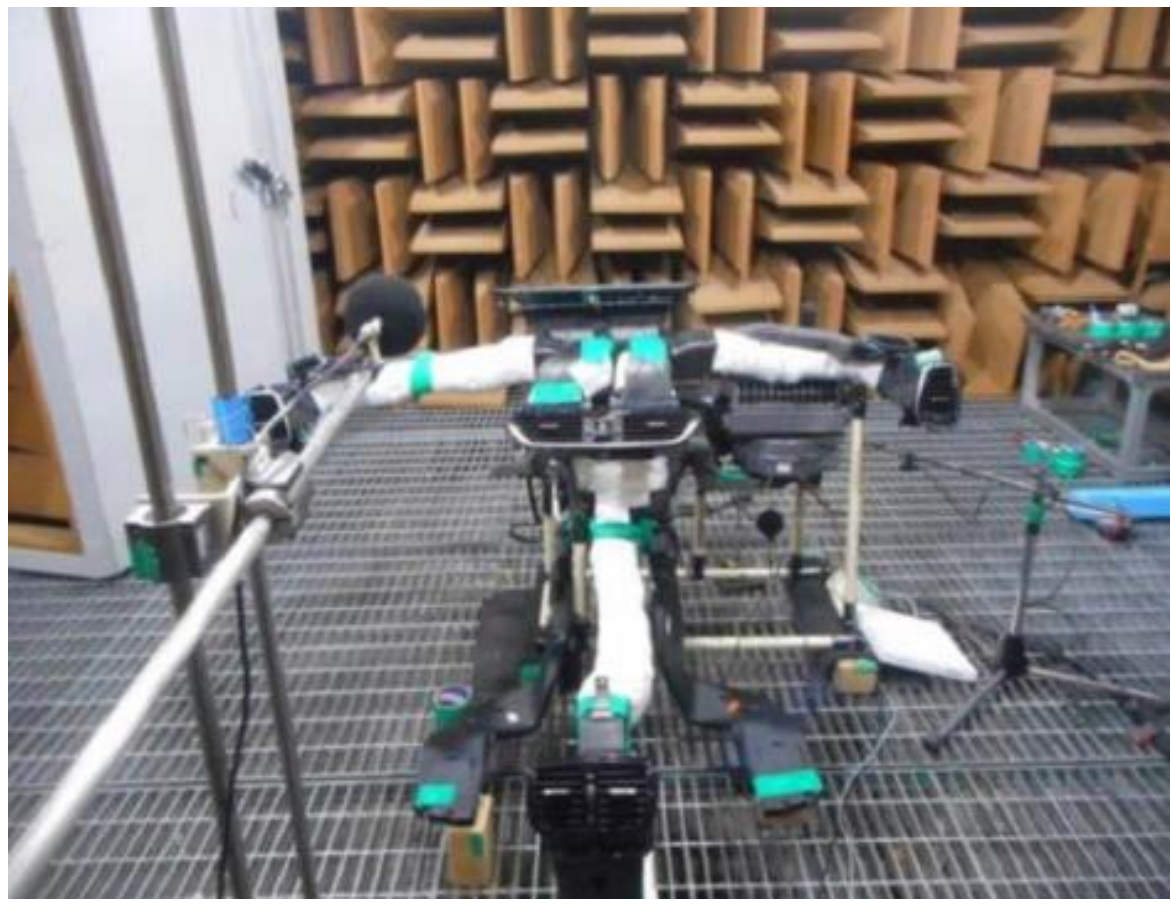

Fig 43. Test Setting in Jung et al. [78] work 


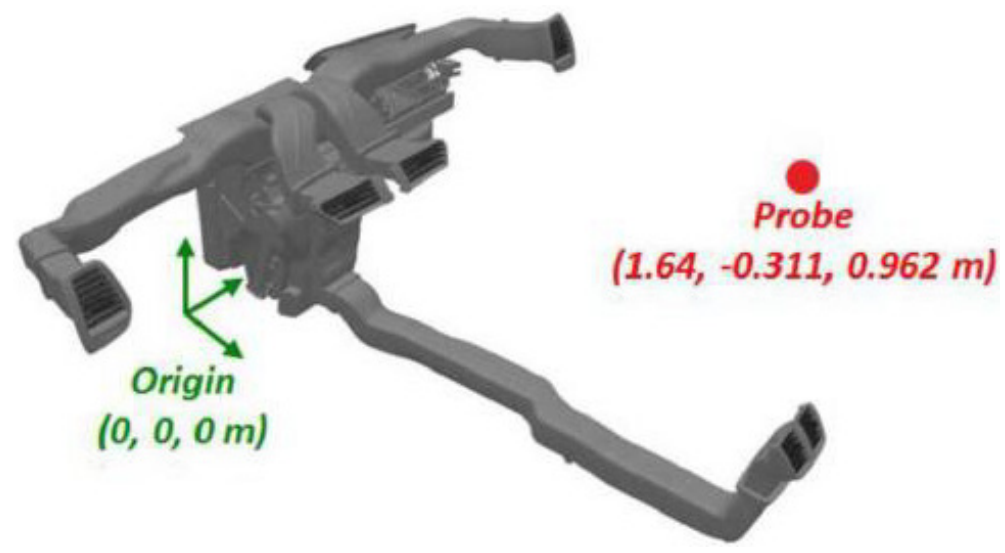

Fig 44. Probe Locations in Jung et al. [78] work

Moreover, Yong-Ju Chu et al. [77] in a recent published work examined the effect of a side mirror on the level of sound generated by an automobile. In this work, two new designs include side mirror with internal and external duct investigated (Fig 42). Their results showed that aerodynamic noise is reduced by using internal duct.

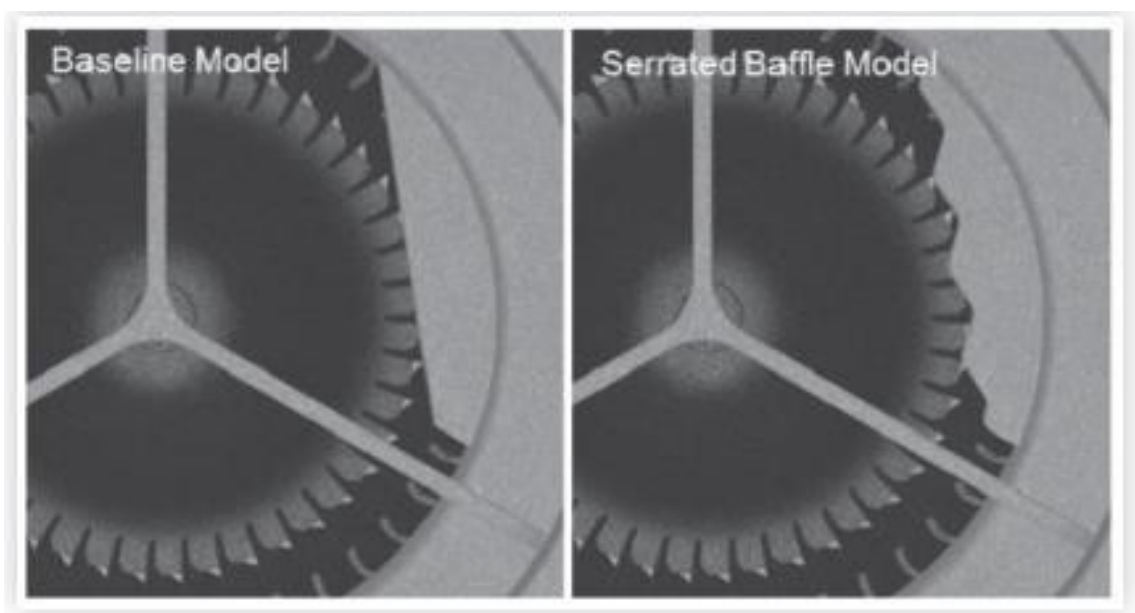

Fig 45. Geometry of HVAC baffle in detail which studied by Jung et al. [78]

Jung et al. [78] investigated the aerodynamic noise of a HVAC unit. In this work, they used the laboratory test stand as shown in Fig 43. In this work, the Lattice-Boltzmann method used for numerical simulation (Fig 44).

In this work, one of the noise reduction approaches that utilize of serrated edges in the HVAC baffle (Fig 45) investigated, and a new design presented according to Fig 46. 


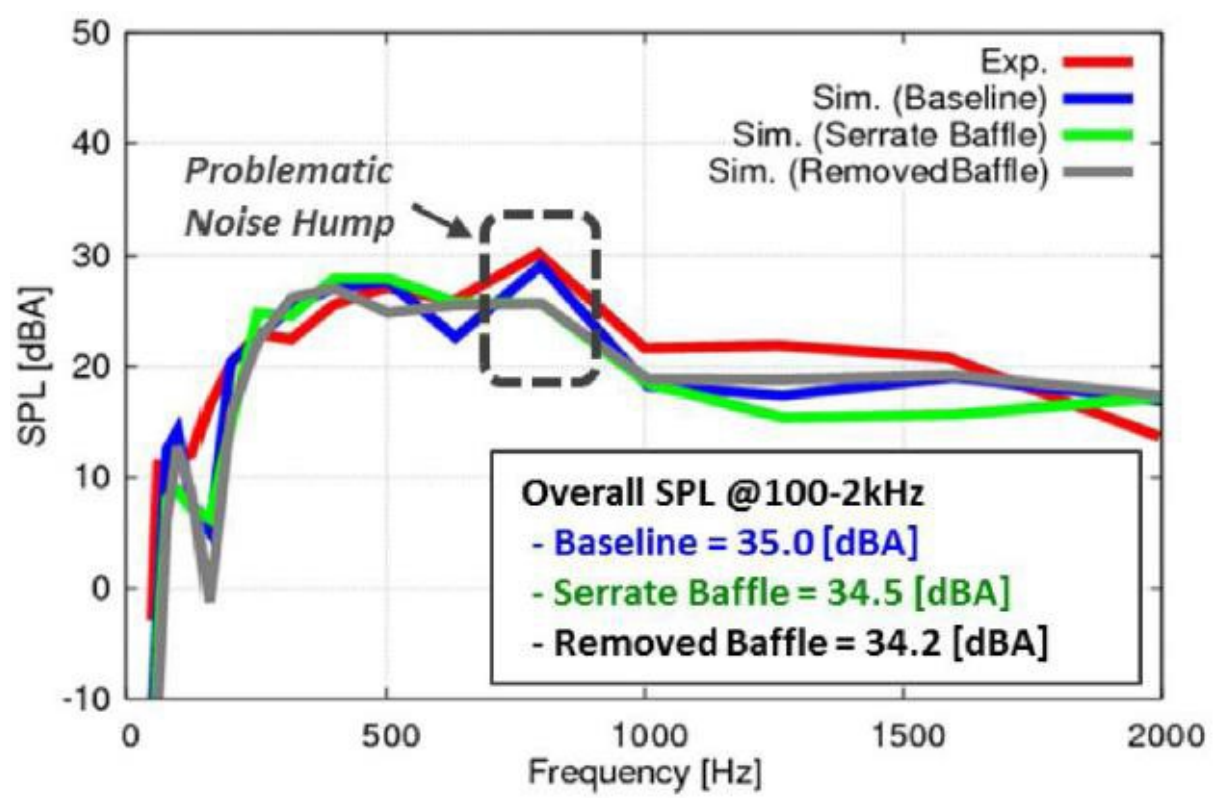

Fig 46. 1/3rd octave band Sound pressure level comparison which studied by Jung et al. [78]

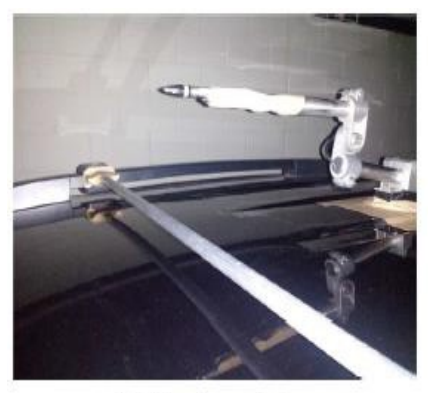

(a) Circular cylinder.

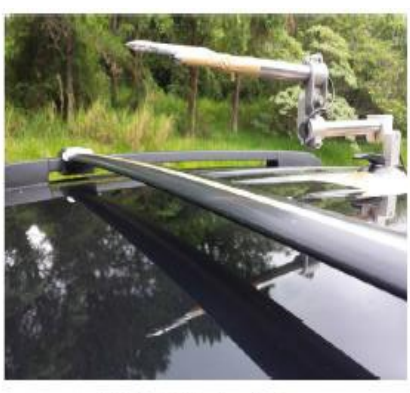

(b) Elliptical cylinder.

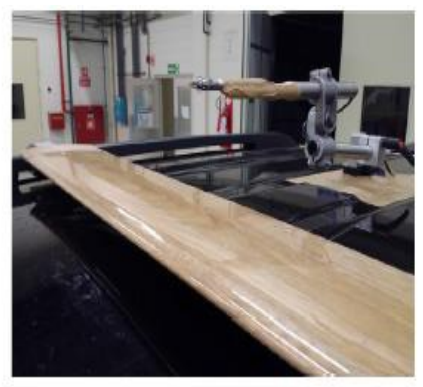

(c) NACA 0012 airfoil.

Fig 47. Crossbar profiles configurations which investigated by Massarotti et al [63]

Massarotti et al [63] investigated the effect of crossbar on aerodynamic noise of vehicle. The work performed experimentally according to Fig 47 and the Reynolds number was between 98,000 and 147,000 . In this work three types of crossbar section include: elliptical, circular and Naca0012 airfoil examined (Fig 48). The results according to Fig 49 showed that the sound level by using airfoil section is much lower than other sections. In this paper, the effect of airfoil angle of attack also investigated. 


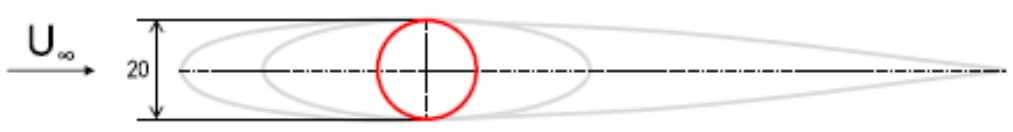

Circular Cylinder

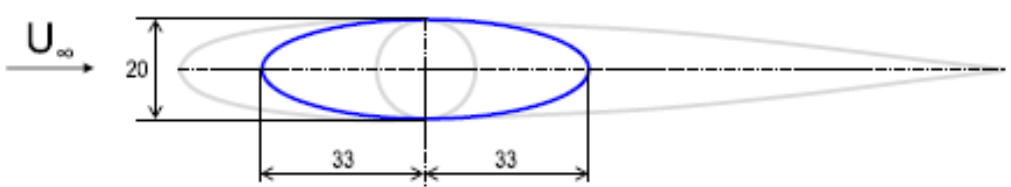

Elliptical profile

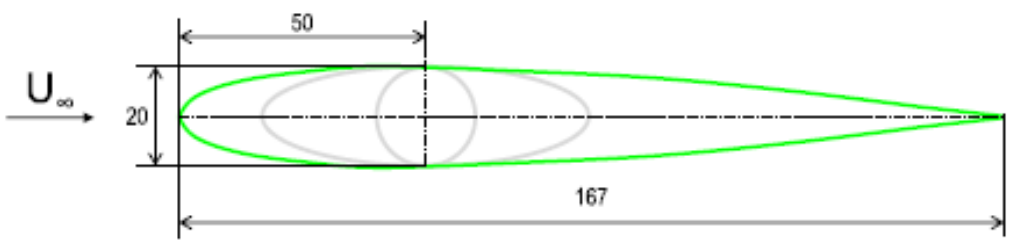

NACA 0012

Fig 48. Different Crossbar section investigated by Massarotti et al [63]

... Circular cylinder - Elliptical cylinder - NACA 0012

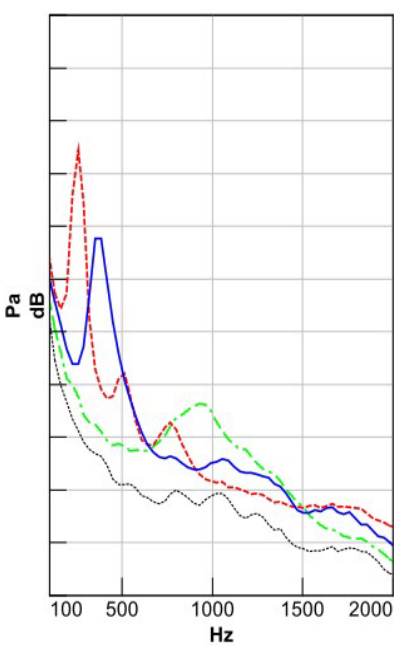

(a) $80 \mathrm{~km} / \mathrm{h}$.

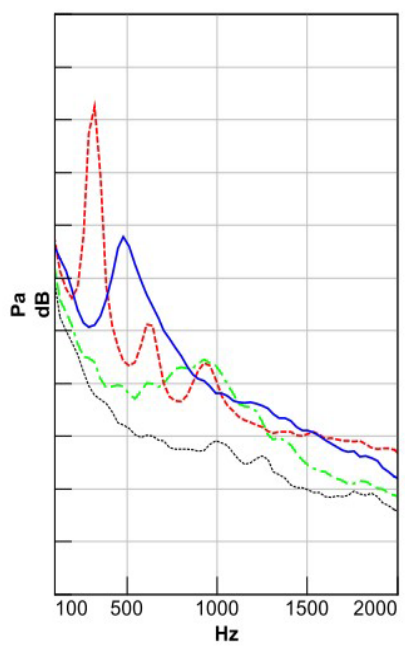

(b) $100 \mathrm{~km} / \mathrm{h}$.

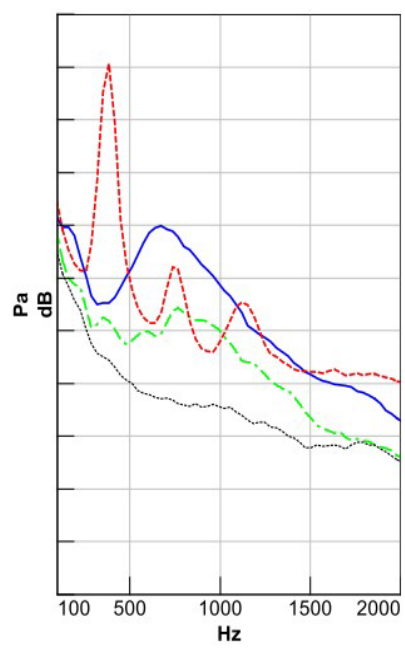

(c) $120 \mathrm{~km} / \mathrm{h}$.

Fig 49. Exterior SPL of different crossbar section profiles for different flow speeds. Which studied by Massarotti et al. [63] 


\section{CONCLUSION}

This paper outlined some applications for noise control in Aerospace, Automotive, and Turbomachinery industries. In this paper critical noise source reviewed, highlights of aero noise control techniques with an emphasis on cutting edge technologies presented along with representative results and relevant conclusions. In each industry, the authors outlined a vision of how aero noise control method could improve industrial products and even could be a response to changing legislation.

The main approach of authors in this review was the synergy of noise control technologies between different industries. It was shown the solutions presented in some industries in other industries were taken into consideration and good results were obtained, for example, the method of edge serration in all three areas, aerospace, turbo machine and automobile had good results in noise reduction. Thus, in each one of these three field, prosper noise reduction method could be proposed and investigated in other fields. The authors recommend, aeroacoustics researchers in each industry first of all focus on the control methods which are effective in other industries because a validated technique is more effective than try and error process with new technique. So a necessary component of any future research effort in each industry should be an appropriately comprehension of aeroacoustics and related technique.

The authors also recommend researchers focus on integrating flow and noise control strategies and the trade-off between them. Since for example a control method in airfoil aero noise reduction could be destructive for aerodynamic characteristics

Finally, for continuing and future work the authors recommend a comprehensive aero noise techniques investigation in any field because aeroacoustics is not limited to the fields presented in this paper and it is also important in rail cars, electronics chip cooling and construction installations, etc.

\section{REFERENCE}

[1] America, A.S. of: PACS 2010 Regular Edition-Acoustics Appendix TO 43: ACOUSTICS. (2013)

[2] Glegg, S., Devenport, W.: Aeroacoustics of low mach number flows: Fundamentals, analysis, and measurement. Academic Press (2017)

[3] ANSI: S1.1-Terminology, American National Standard: Acoustic. Sec 3.03. (1994)

[4] Spalart, P.R., Shur, M.L., Strelets, M.K., Travin, A.K.: Initial noise predictions for rudimentary landing gear. J. Sound Vib. 330, 4180-4195 (2011)

[5] Ffowcs Williams, J.E., Hawkings, D.L.: Sound generation by turbulence and surfaces in arbitrary motion. Philos. Trans. R. Soc. London. Ser. A, Math. Phys. Sci. 264, 321-342 (1969)

[6] Lighthill, M.J.: On sound generated aerodynamically I. General theory. Proc. R. Soc. London. Ser. A. Math. Phys. Sci. 211, 564-587 (1952)

[7] Lighthill, M.J.: On sound generated aerodynamically II. Turbulence as a source of sound. Proc. R. Soc. London. Ser. A. Math. Phys. Sci. 222, 1-32 (1954)

[8] Atassi, O., Gilson, J.J.: IUTAM Symposium on Computational Aero-Acoustics for Aircraft Noise Prediction Future Challenges in Computational Aeroacoustics for Fan Broadband and Combustor Noise. Procedia Eng. 6, 193-202 (2010).

[9] Krishna, S.R., Krishna, A.R., Ramji, K.: Reduction of motor fan noise using CFD and CAA simulations. Appl. Acoust. 72, 982-992 (2011). 
[10] Pérez Arroyo, C., Kholodov, P., Sanjosé, M., Moreau, S.: CFD modeling of a realistic turbofan blade for noise prediction. Part 1: Aerodynamics. (2019)

[11] Ando, S., Kimura, K., Segawa, K., Yamamoto, K.: Study on the Hybrid Method of CFD and Bubble Dynamics for Marine Propeller Cavitation Noise Prediction. In: Proceedings of the 10th International Symposium on Cavitation (CAV2018). ASME Press (2018)

[12] Tester, B., Powles, C., Baker, N.J., Kempton, A.J.: Scattering of sound by liner splices: a Kirchhoff model with numerical verification. AIAA J. 44, 2009-2017 (2006)

[13] Hardin, J.C., Lamkin, S.L.: Aeroacoustic Computation of Cylinder Wake Flow. AIAA J. 22, 51-57 (1984)

[14] Hardin, J.C., Lamkin, S.L.: Computational Aeroacoustics-Present Status and Future Promise. In: Aero-and Hydro-Acoustics. pp. 253-259. Springer (1986)

[15] Niemöller, A., Meinke, M.H., Schroeder, W., Albring, T.A., Gauger, N.R.: Noise Reduction Using a Direct-Hybrid CFD/CAA Method. 1-15 (2019).

[16] Gutin, L.: On the sound field of a rotating propeller. (1948)

[17] Curle, N.: The influence of solid boundaries upon aerodynamic sound. Proc. R. Soc. London. Ser. A. Math. Phys. Sci. 231, 505-514 (1955)

[18] Tam, C.K.W.: Computational aeroacoustics-Issues and methods. AIAA J. 33, 1788-1796 (1995)

[19] Gély, D., Leylekian, L.: Aeroacoustics: an Overview for Air Vehicle Applications. (2014)

[20] ICAO, I.: Aircraft Engine Emissions Databank. Int. Civ. Aviat. Organ. (2006)

[21] ICAO, I.: Aircraft Engine Emissions Databank. Int. Civ. Aviat. Organ. (2002)

[22] Huff, D.L.: Noise reduction technologies for turbofan engines. (2007)

[23] Hartl, D.J., Mooney, J.T., Lagoudas, D.C., Calkins, F.T., Mabe, J.H.: Use of a Ni60Ti shape memory alloy for active jet engine chevron application: II. Experimentally validated numerical analysis. Smart Mater. Struct. 19, 15021 (2009)

[24] Mabe, J., Calkins, F., Butler, G.: Boeing's variable geometry chevron, morphing aerostructure for jet noise reduction. In: 47th AIAA/ASME/ASCE/AHS/ASC Structures, Structural Dynamics, and Materials Conference 14th AIAA/ASME/AHS Adaptive Structures Conference 7th. p. 2142 (2006)

[25] Calkins, F., Butler, G.: Subsonic jet noise reduction variable geometry chevron. In: 42nd AIAA Aerospace Sciences Meeting and Exhibit. p. 190 (2004)

[26] Papamoschou, D., Shupe, R.: Effect of nozzle geometry on jet noise reduction using fan flow deflectors. In: 12th AIAA/CEAS Aeroacoustics Conference (27th AIAA Aeroacoustics Conference). p. 2707 (2006)

[27] Papamoschou, D., Nishi, K.: Jet noise suppression with fan flow deflectors in realisticshaped nozzle. In: 43rd AIAA Aerospace Sciences Meeting and Exhibit. p. 993 (2005)

[28] Papamoschou, D.: Parametric study of fan flow deflectors for jet noise suppression. In: 11 th AIAA/CEAS Aeroacoustics Conference. p. 2890 (2005)

[29] Seiner, J.M., Gilinsky, M.M.: Nozzle thrust optimization while reducing jet noise. AIAA J. 35, 420-427 (1997)

[30] Narayanan, V.: Computational study of noise reduction in subsonic jet using chevron nozzles, (2019) 
[31] Varghese, J.T., NS, S.R., Shafi, M.S.: Analysis of Acoustic Testing on Static Chevrons Nozzles. In: 2019 Advances in Science and Engineering Technology International Conferences (ASET). pp. 1-6. IEEE (2019)

[32] Engblom, W., Khavaran, A., Bridges, J.: Numerical prediction of chevron nozzle noise reduction using WIND-MGBK methodology. In: 10th AIAA/CEAS Aeroacoustics Conference. p. 2979 (2004)

[33] Kanmaniraja, R., Freshipali, R., Abdullah, J., Niranjan, K., Balasubramani, K., Kumar, V.R.S.: 3D numerical studies on jets acoustic characteristics of chevron nozzles for aerospace applications. Int. Sch. Sci. Res. Innov. 8, 1510-1516 (2014)

[34] Jaworski, J.W., Peake, N.: Aerodynamic noise from a poroelastic trailing edge with implications for the silent flight of owls. 18th AIAA/CEAS Aeroacoustics Conf. (33rd AIAA Aeroacoustics Conf. 4-6 (2012).

[35] Gruber, M., Joseph, P.F., Polacsek, C., Chong, T.P.: Noise reduction using combined trailing edge and leading edge serrations in a tandem airfoil experiment. 18th AIAA/CEAS Aeroacoustics Conf. (33rd AIAA Aeroacoustics Conf. 4-6 (2012).

[36] Strawn, R.C., Biswas, R.: Numerical simulations of helicopter aerodynamics and acoustics. J. Comput. Appl. Math. 66, 471-483 (1996).

[37] Appelbaum, J., Duda, B., Fares, E., Khorrami, M.R.: Airframe noise simulations of a fullscale aircraft. 2018 AIAA/CEAS Aeroacoustics Conf. 1-15 (2018).

[38] Arcondoulis, E., Liu, Y., Li, Z., Yang, Y., Wang, Y., Li, W.: The design and noise characteristics of a structured porous coated cylinder in uniform flow. In: 2018 AIAA/CEAS Aeroacoustics Conference. American Institute of Aeronautics and Astronautics, Reston, Virginia (2018)

[39] Biedermann, T.M., Czeckay, P., Geyer, T.F., Kameier, F., Paschereit, C.O.: Noise source identification of aerofoils subjected to leading edge serrations using phased array beamforming. 2018 AIAA/CEAS Aeroacoustics Conf. 1-18 (2018).

[40] Chirico, G., Barakos, G.N., Bown, N.: Numerical aeroacoustic analysis of propeller designs. Aeronaut. J. 122, 283-315 (2018).

[41]Li, L., Liu, P., Guo, H., Geng, X., Hou, Y., Wang, J.: Aerodynamic and aeroacoustic experimental investigation of 30P30N high-lift configuration. Appl. Acoust. 132, 43-48 (2018).

[42] Takaishi, T., Kumada, T., Yokokawa, Y., Ito, Y., Murayama, M., Sakai, R., Shimada, A., Yamamoto, K.: Further noise reduction design for landing gear toward FQUROH second flight demonstration. 2018 AIAA/CEAS Aeroacoustics Conf. 1-16 (2018).

[43] Meher-Homji, C.B.: The Historical Evolution Of Turbomachinery. In: Proceedings of the 29th Turbomachinery Symposium. Texas A\&M University. Turbomachinery Laboratories (2000)

[44] Arnold, B., Lutz, T., Krämer, E.: Design of a boundary-layer suction system for turbulent trailing-edge noise reduction of wind turbines. Renew. Energy. 123, 249-262 (2018).

[45] Arakawa, C., Fleig, O., Iida, M., Shimooka, M.: Numerical approach for noise reduction of wind turbine blade tip with earth simulator. J. Earth Simulator. 2, 11-33 (2005)

[46] Ghasemian, M., Nejat, A.: Aero-acoustics prediction of a vertical axis wind turbine using Large Eddy Simulation and acoustic analogy. Energy. 88, 711-717 (2015)

[47] Kuntz, M., Lohmann, D., Lieser, J.A., Pahlke, K.: Comparison of Rotor Noise Predictions by a Lifting Surface Method and Euler Solutions Using Kirchhoff Equation. In: Proc. First Joint CEAS/AQIAA Aeroacoustic Conf. 1995 (1995) 
[48] Lieser, J.A., Lohmann, D., Rohardt, C.H.: Aeroacoustic Design of a 6-Bladed Propeller. Aerosp. Sci. Technol. 1, 381-389 (1997).

[49] Fehse, K.-R., Neise, W.: Generation mechanisms of low-frequency centrifugal fan noise. AIAA J. 37, 1173-1179 (1999)

[50] Jones, B.R., Crossley, W.A., Lyrintzis, A.S.: Aerodynamic and aero acoustic optimization of airfoils via a parallel genetic algorithm. 7th AIAA/USAF/NASA/ISSMO Symp. Multidiscip. Anal. Optim. 37, (1998).

[51] Chapman, C.J.: Some benchmark problems for computational aeroacoustics. J. Sound Vib. 270, 495-508 (2004)

[52] Kim, T., Lee, S., Kim, H., Lee, S.: Design of low noise airfoil with high aerodynamic performance for use on small wind turbines. Sci. China Ser. E Technol. Sci. 53, 75-79 (2010)

[53] Mohamed, M.H.: Aero-acoustics noise evaluation of H-rotor Darrieus wind turbines. Energy. 65, 596-604 (2014).

[54] Mohamed, M.H.: Reduction of the generated aero-acoustics noise of a vertical axis wind turbine using CFD (Computational Fluid Dynamics) techniques. Energy. 96, 531-544 (2016)

[55] Maizi, M., Mohamed, M.H., Dizene, R., Mihoubi, M.C.: Noise reduction of a horizontal wind turbine using different blade shapes. Renew. Energy. 117, 242-256 (2018).

[56] Cerrato, G.: Automotive sound quality-powertrain, road and wind noise. Sound Vib. 43, 16-24 (2009)

[57] Murata, H., Tanaka, H., Takada, H., Ohsasa, Y.: Sound quality evaluation of passenger vehicle interior noise. SAE Technical Paper (1993)

[58] Brandl, F.K., Biermayer, W.: A new tool for the onboard objective assessment of vehicle interior noise quality. SAE Technical Paper (1999)

[59] Gonzalez, A., Ferrer, M., De Diego, M., Pinero, G., Garcia-Bonito, J.J.: Sound quality of low-frequency and car engine noises after active noise control. J. Sound Vib. 265, 663679 (2003)

[60] Mohamed, E.S., Abouel-Seoud, S., Eltantawie, M., Mohamded, A., Salah, M.: Improved vehicle interior structure-borne noise induced by the powertrain using parallel dry friction damper. J. Low Freq. Noise, Vib. Act. Control. 37, 295-312 (2018)

[61]Hucho, W., Sovran, G.: Aerodynamics of road vehicles. Annu. Rev. Fluid Mech. 25, 485$537(1993)$

[62] Musser, C., Calloni, M., Golota, A., Zerbib, N.: Aero-Vibro-Acoustic Simulation Methodologies for Vehicle Wind Noise Reduction. SAE Technical Paper (2019)

[63] Massarotti, M.R., Wolf, W.R.: Aeroacoustic analysis of automotive roof crossbars through on-track acoustic measurements. Appl. Acoust. 142, 95-105 (2018).

[64] Gloerfelt, X.: Noise from automotive components. Aerodyn. noise from wall-bounded flows. 9-13 (2009)

[65]Hartmann, M., Ocker, J., Lemke, T., Mutzke, A., Schwarz, V., Tokuno, H., Toppinga, R., Unterlechner, P., Wickern, G.: Wind noise caused by the side-mirror and a-pillar of a generic vehicle model. In: 18th AIAA/CEAS Aeroacoustics Conference (33rd AIAA Aeroacoustics Conference). p. 2205 (2012) 
[66] Tsai, C.H., Fu, L.M., Tai, C.H., Huang, Y.L., Leong, J.C.: Computational aero-acoustic analysis of a passenger car with a rear spoiler. Appl. Math. Model. 33, 3661-3673 (2009).

[67] Shojaefard, M.H., Goudarzi, K., Fotouhi, H.: Numerical study of airflow around vehicle a- pillar region and windnoise generation prediction. Am. J. Appl. Sci. 6, 276 (2009)

[68] Wu, S.F., Su, S.G., Shah, H.S.: Modelling of the noise spectra of axial flow fans in a free field. J. Sound Vib. 200, 379-399 (1997)

[69] Vad, J.: Forward blade sweep applied to low-speed axial fan rotors of controlled vortex design: an overview. J. Eng. Gas Turbines Power. 135, 12601 (2013)

[70] Kalmár-nagy, T., Bak, B.D.: Vibration and Noise of an Axial Flow Fan. 109-113 (2015).

[71]Benedek, T., Tóth, P.: Beamforming measurements of an axial fan in an industrial environment. Period. Polytech. Mech. Eng. 57, 37-46 (2013)

[72] Underbrink, J.R.: Aeroacoustic phased array testing in low speed wind tunnels. In: Aeroacoustic measurements. pp. 98-217. Springer (2002)

[73] Sijtsma, P., Oerlemans, S., Holthusen, H.: Location of rotating sources by phased array measurements. In: 7th AIAA/CEAS Aeroacoustics Conference and Exhibit. p. 2167 (2001)

[74] De Gennaro, M., Kuehnelt, H.: Broadband noise modelling and prediction for axial fans. In: Proceedings of the Internataional Conference Fan Noise, Technology and Numerical Methods (2012)

[75]Bennouna, S., Matharan, T., Cheriaux, O.: Automotive HVAC Noise Reduction. SAE Tech. Pap. 2018-June, 1-5 (2018).

[76] Chen, X., Wang, S., Wu, Y. qiang, Li, Y. yang, Wang, H. yu: Experimental and numerical investigations of the aerodynamic noise reduction of automotive side view mirrors. J. Hydrodyn. 30, 642-650 (2018).

[77] Chu, Y.-J., Shin, Y.-S., Lee, S.-Y.: Aerodynamic analysis and noise-reducing design of an outside rear view mirror. Appl. Sci. 8, 519 (2018)

[78] Jung, W., Cha, Y., Song, S., Byun, S., Ho, S., Kim, M.: Direct Aeroacoustics Predictions of Automotive HVAC Systems based on Lattice Boltzmann Method. SAE Tech. Pap. 2018-June, 1-7 (2018). 